\title{
The Chapman-Enskog Expansion and the Quantum Hydrodynamic Model for Semiconductor Devices
}

\author{
CARL L. GARDNER ${ }^{*, \dagger}$ and CHRISTIAN RINGHOFER* \\ Department of Mathematics, Arizona State University, Tempe, AZ 85287-1804
}

(Received 16 December 1998; In final form 14 December 1999)

\begin{abstract}
A "smooth" quantum hydrodynamic (QHD) model for semiconductor devices is derived by a Chapman-Enskog expansion of the Wigner-Boltzmann equation which can handle in a mathematically rigorous way the discontinuities in the classical potential energy which occur at heterojunction barriers in quantum semiconductor devices. A dispersive quantum contribution to the heat flux term in the QHD model is introduced.
\end{abstract}

Keywords: Quantum hydrodynamic model, moment expansion

\section{INTRODUCTION}

This investigation is concerned with the derivation of a quantum hydrodynamic (QHD) model in the presence of discontinuities in the classical potential energy which occur at heterojunction barriers in quantum semiconductor devices. In general, QHD models describe the motion of an ensemble of charged particles (electrons) in the mean field approximation and are of the form

$$
\begin{gathered}
\partial_{t} n+\nabla_{x} \cdot(n u)=0, \\
\partial_{t}(m n u)+\nabla_{x} \cdot\left[P+m n u u^{T}\right]+e n \nabla_{x} V^{\text {tot }}+\frac{m n u}{\tau_{p}}=0,
\end{gathered}
$$

$$
\begin{gathered}
\partial_{t}\left(w+m|u|^{2} n\right)+\nabla_{x} \cdot\left[q+w u+2 P u+m u|u|^{2} n\right] \\
+2 e n\left(\nabla_{x} V^{\mathrm{tot}}\right) \cdot u \\
+\frac{w+m|u|^{2} n-3 n T_{0}}{\tau_{w}}=0 \\
V^{\mathrm{tot}}=V^{M}+V^{B}
\end{gathered}
$$

where the particle ensemble is described by its density $n$, its group velocity $u$, and its internal energy density $w$. (Note that $w$ here is twice the usual internal energy density.) Equations (1)-(3) give the conservation of particles, momentum, and energy, respectively. $V^{M}$ is the mean field potential which is computed from Poisson's equation. We will, however, for the purposes of this paper,

* Research supported in part by the National Science Foundation under grants DMS-9706792 and INT-9603253.

${ }^{\dagger}$ Corresponding author. Tel.: 602-965-0226, Fax: 602-965-0461, e-mail: gardner@asu.edu 
assume $V^{M}$ to be given and ignore the self-consistent coupling. In the presence of interfaces between different materials, this mean field potential is calibrated by the barrier potential $V^{B}$ which models the gap between the conduction bands of each material. Thus $V^{B}$ is a given function which is constant inside each material. The constants $m$ and $e$ denote the effective mass and the charge of the electron. Boltzmann's constant $k_{B}$ has been set to 1. $\tau_{p}$ and $\tau_{w}$ denote the relaxation times for momentum and energy, and $T_{0}$ is the ambient temperature.

In order to close the hydrodynamic system the remaining variables (the stress tensor $P$ and the heat flux q) have to be expressed in terms of the primary variables $n, u, w$ and $V$. For the classical hydrodynamic system this closure is obtained through the Maxwellian assumption [1], giving $q=0$ and $P=(w / 3) I$. In the quantum case the first two conservation laws (1) and (2) can be derived directly from a nonlinear Schrödinger equation. In this case one obtains an explicit formula for the internal energy density $w$ in terms of $n$, depending on the form of interaction potential used in the Hamiltonian of the Schrödinger equation [2]. Alternatively, one can start from the Wigner-Boltzmann equation

$$
\begin{aligned}
& \partial_{t} F+\frac{1}{m} \nabla_{x} \cdot(p F) \\
& -\frac{i e}{\hbar}\left[V^{\operatorname{tot}}\left(x+\frac{\hbar}{2 i} \nabla_{p}\right)-V^{\operatorname{tot}}\left(x-\frac{\hbar}{2 i} \nabla_{p}\right)\right] F \\
& \quad+Q(F)=0,
\end{aligned}
$$

using a more or less phenomenological collision operator $Q$, and derive the system directly by computing the moments of the Wigner (distribution) function $F(x, p, t)$. Closure (expressions for $P$ and $q$ in terms of $n, u$, and $w$ ) is then obtained by using an appropriately parametrized thermal equilibrium distribution function. We introduce the notation

$$
\begin{aligned}
M^{0}(F) & =\int F d p, \quad M_{j}^{1}(F)=\int p_{j} F d p \\
M_{j k}^{2}(F) & =\int p_{j} p_{k} F d p
\end{aligned}
$$

$$
\begin{gathered}
M_{j}^{3}(F)=\int p_{j}|p|^{2} F d p \\
M_{j k}^{4}(F)=\int p_{j} p_{k}|p|^{2} F d p \\
\operatorname{trM}^{2}(F)=\int|p|^{2} F d p, \quad t r M^{4}(F)=\int|p|^{4} F d p
\end{gathered}
$$

for the moments of a function $F$ with respect to the momentum vector $p$. $\left(M^{1}\right.$ and $M^{3}$ are vectors and $M^{2}$ and $M^{4}$ are $3 \times 3$ matrices.) Here the symbol $t r$ denotes the usual trace of a matrix. The QHD equations are obtained by taking the first three moments $\left(M^{0}, M^{1}, t r M^{2}\right)$ of Eq. (5) and by expressing the moments of the Wigner function $F$ in terms of center of mass coordinates. If we define $n$ as the zero order moment of the Wigner function $F$ and the mean velocity by $n=M^{0} F$, $m n u=M^{1} F$, and define the centered distribution $F^{\prime}$ by $F^{\prime}(x, p, t)=F(x, p+m u, t)$, we obtain the QHD system (1)-(3) where the variables are now defined by the moments of $F$ and $F^{\prime}$ :

$$
\begin{gathered}
n=M^{0} F, \quad u=\frac{1}{m n} M^{1} F, \quad P=\frac{1}{m} M^{2} F^{\prime}, \\
w=\frac{1}{m} t r M^{2} F^{\prime}=t r P, \quad q=\frac{1}{m^{2}} M^{3} F^{\prime},
\end{gathered}
$$

and the collision operator $Q$ is assumed to be of such a form that its moments satisfy

$$
\begin{aligned}
M^{0} Q & =0, \quad M^{1} Q=\frac{m n}{\tau_{p}} u \\
t r M^{2} Q & =\frac{m w+m^{2}|u|^{2} n-3 m n T_{0}}{\tau_{w}} .
\end{aligned}
$$

Thus the closure problem for the QHD equations can be formulated as expressing the shifted moments $M^{2} F^{\prime}$ and $M^{3} F^{\prime}$ of the Wigner function $F$ in equation (5) in terms of the primary variables $M^{0} F, M^{1} F$, and $\operatorname{tr} M^{2} F^{\prime}$. We will derive expressions for $P$ and $q$ in terms of the primary variables $n, u$, and $w$ via a Chapman-Enskog expansion of the Wigner-Boltzmann equation (5) using a BGK type collision operator which conserves particles and relaxes momentum and energy according to the relaxation times $\tau_{p}$ and $\tau_{w}$. The basic assumption 
here is that the momentum relaxation time $\tau_{p}$ is much smaller than the energy relaxation time $\tau_{w}$. The expansion will use the small dimensionless parameter $\tau=\tau_{p} / \tau_{w}$. Furthermore we consider the Wigner-Boltzmann equation in a moderately high field regime, meaning that differences in the mean field potential $V^{M}$ dominate differences in the barrier potential $V^{B}$, but that collisions are still the strongest mechanism in Eq. (5). The results of this procedure are closure conditions of the form

$$
\begin{aligned}
& P=\frac{w}{3} I+\hbar^{2}\left(P^{Q 1}+P^{Q 2}\right), \\
& q=-\frac{5 \tau_{p} w}{9 m} \nabla_{x}\left(\frac{w}{n}\right)-\frac{\hbar^{2} n}{4 m} \Delta_{x} u,
\end{aligned}
$$

where the quantum corrections to the stress tensor $P$ are determined by the equilibrium distribution function used in the BGK operator. An approximate form of the equilibrium distribution function is derived in Section 3 via a Chapman-Enskog type expansion of the Bloch equation [3], which determines the thermal equilibrium distribution function for a thermalized quantum system of Boltzmann distributed states. The quantum corrections $P^{Q 1}$ and $P^{Q^{2}}$ are given by

$$
\begin{gathered}
P_{j k}^{Q 1}=\frac{n^{2}}{4 m w}\left(\partial_{x_{j k}} H-\frac{1}{3} \delta_{j k} \Delta_{x} H\right), \\
H=\frac{w}{3 n}\left[-\ln n+\frac{3}{2} \ln \left(\frac{2 \pi m w}{3 n \hbar^{2}}\right)\right], \\
P_{j k}^{Q 2}=\frac{e n^{2}}{4 m w}\left\{3 \partial_{x_{j k}}-\delta_{j k} \Delta_{x}\right\} \\
\Gamma_{2}\left(\frac{\hbar}{2 i \sqrt{(m w / 3 n)}} \nabla_{x}\right) V^{B}(x), \\
\Gamma_{2}(\xi)=\int_{0}^{1} \gamma^{2} \exp \left[\frac{|\xi|^{2}}{2}\left(\gamma^{2}-1\right)\right] d \gamma,
\end{gathered}
$$

where the term $\Gamma_{2} V^{B}$ is a smoothed version of the barrier potential. It is a relatively easy exercise to show that the symbol of the pseudo-differential operator $\Gamma_{2}$ decays like $|\xi|^{-2}$ for large $\xi$. Therefore the second partial derivatives of $\Gamma_{2} V^{B}$ have essentially the same discontinuities as the original barrier potential $V^{B}$.

The formulas (12)-(15) close the QHD system and a solution can be computed in terms of the primary variables $n, u$, and $w$. Due to physical considerations it is important to define a temperature $T$ by adding an equation of state relating the internal energy density $w$ to the temperature. Using the same Chapman-Enskog expansion of the Bloch equation, the equation of state can be derived (albeit with a slight inconsistency, explained in Section 3). It is of the form

$$
\begin{aligned}
w= & 3 n T-\frac{\hbar^{2} n}{12 m T} \Delta_{x}\left\{T \ln \left[n\left(\frac{\hbar^{2}}{2 \pi m T}\right)^{3 / 2}\right]\right\} \\
& +\frac{e \hbar^{2} n}{4 m T} \Delta_{x} \Gamma_{2}\left(\frac{\hbar}{2 i \sqrt{m T}} \nabla_{x}\right) V^{B}(x) .
\end{aligned}
$$

Neglecting $O\left(\hbar^{4}\right)$ and $O\left(\tau_{p} \hbar^{2}\right)$ terms, the stress tensor $P$ and the heat flux $q$ are then given in terms of the temperature as

$$
\begin{gathered}
P=n T \delta_{j k}+\hbar^{2}\left(P^{Q 1^{\prime}}+P^{Q 2^{\prime}}\right), \\
q=-\frac{5 \tau_{p} n T}{m} \nabla_{x} T-\frac{\hbar^{2} n}{4 m} \Delta_{x} u \\
P_{j k}^{Q 1^{\prime}}=\frac{n}{12 m T} \partial_{x_{j k}} T \ln \left[\frac{1}{n}\left(\frac{2 \pi m T}{\hbar^{2}}\right)^{3 / 2}\right], \\
P_{j k}^{Q 2^{\prime}}=\frac{e n}{4 m T} \partial_{x_{j k}} \Gamma_{2}\left(\frac{\hbar}{2 i \sqrt{m T}} \nabla_{x}\right) V^{B}(x) .
\end{gathered}
$$

Thus the resulting QHD system, using the equation of state (16), is of the form

$$
\begin{gathered}
\partial_{t} n+\nabla_{x} \cdot(n u)=0, \\
\partial_{t}(m n u)+\nabla_{x}(n T)+\nabla_{x} \cdot\left[m n u u^{T}+\hbar^{2}\left(P^{Q 1^{\prime}}+P^{Q 2^{\prime}}\right)\right] \\
+e n \nabla_{x} V^{\mathrm{tot}}+\frac{m n u}{\tau_{p}}=0 \\
\partial_{t}\left(w+m|u|^{2} n\right) \\
+\nabla_{x} \cdot\left[-\frac{5 \tau_{p} n T}{m} \nabla_{x} T-\frac{\hbar^{2} n}{4 m} \Delta_{x} u+w u\right. \\
+2 n T u+2 \hbar^{2}\left(P^{Q 1^{\prime}}+P^{Q 2^{\prime}}\right) u \\
\left.+m u|u|^{2} n\right] \\
+2 e n\left(\nabla_{x} V^{\mathrm{tot}}\right) \cdot u \\
+\frac{w+m|u|^{2} n-3 n T_{0}}{\tau_{w}}=0
\end{gathered}
$$




$$
\begin{gathered}
w=3 n T-\frac{\hbar^{2} n}{12 m T} \Delta_{x}\left\{T \ln \left[n\left(\frac{\hbar^{2}}{2 \pi m T}\right)^{3 / 2}\right]\right\} \\
+\frac{e \hbar^{2} n}{4 m T} \Delta_{x} \Gamma_{2}\left(\frac{\hbar}{2 i \sqrt{m T}} \nabla_{x}\right) V^{B}(x) \\
V^{\text {tot }}=V^{M}+V^{B} .
\end{gathered}
$$

This QHD system is novel in several respects. First, note that the heat flux $q$ contains in addition to the expected heat conduction term of the form $-n T \nabla_{x} T$ a dispersive quantum correction depending on the second derivative of the velocity (derived originally in Ref. [4] in a very different way).

Second, note that the first quantum correction $P^{Q 1^{\prime}}$ of the stress tensor in Eq. (18) reduces up to a constant factor to the term given by the Bohm potential [2] if the temperature is held constant. One result of the Chapman-Enskog expansion of the equilibrium distribution function is the temperature dependence of this potential. The second term $P^{Q 2^{\prime}}$ is essentially the smoothed potential approximation derived in Refs. [5] and [6]. However, while the approximations there (closure of the system by means of a momentum displaced equilibrium distribution function) led to a hyperbolic transport system with a parabolic heat flux term, the new closure, which is based on an asymptotic analysis of the transient problem, leads to a dispersive heat flux term.

Below in Section 2 we derive the approximation of the Wigner-Boltzmann equation for a general BGK operator, for which we only use the moment balance corresponding to the given relaxation time. This determines the QHD equations up to the form of the quantum corrections in the stress tensor. In Section 3 we derive expressions for $P^{Q^{1}}$ and $P^{Q 2}$ from the Bloch equation.

\section{THE CHAPMAN-ENSKOG EXPANSION OF THE WIGNER-BOLTZMANN EQUATION}

In this section we will derive the closure conditions for the quantum hydrodynamic model (i.e., the expressions for the stress tensor $P$ and the heat flux $q$ ) from a Chapman-Enskog type expansion of the Wigner-Boltzmann equation. As a collision term we will assume a BGK type operator that relaxes the solution against an equilibrium distribution function $\Omega$ which is parametrized by its moments $M^{0} \Omega$ and $\operatorname{tr} M^{2} \Omega$. The shape of the closure terms $P$ and $q$ in Eqs. (2) and (3) is determined by the functional form of this equilibrium distribution function. The form for $\Omega$ is then discussed in the next section. The collisionless Wigner-Boltzmann equation is given by

$$
\begin{gathered}
\partial_{t} F+\frac{1}{m} \nabla_{x} \cdot(p F)-\Theta\left[V+V^{B}\right] F=0, \\
\Theta[V]=\frac{i e}{\hbar}\left[V\left(x+\frac{\hbar}{2 i} \nabla_{p}\right)-V\left(x-\frac{\hbar}{2 i} \nabla_{p}\right)\right],
\end{gathered}
$$

where the operator $\Theta$ is defined in the usual sense of pseudo-differential operators via the Fourier transform of $F$. It is a calculus exercise to verify that Eq. (25) is equivalent to the Schrödinger equation for mixed states. That is, if the wave function $\psi_{j}$ for each pure state satisfies the Schrödinger equation

$$
\partial_{t} \psi_{j}=\frac{i \hbar}{2 m} \Delta_{x} \psi_{j}-\frac{i e}{\hbar}\left(V+V^{B}\right) \psi_{j}
$$

then the Wigner function for the mixed state defined by

$$
\begin{array}{r}
F(x, p, t)=\sum_{j} a_{j} \int \psi\left(x-\frac{\hbar}{2} \eta\right) \\
\psi^{*}\left(x+\frac{\hbar}{2} \eta\right) e^{i \eta \cdot p} d \eta
\end{array}
$$

satisfies the Wigner equation (25). Here the $a_{j}$ 's denote occupation numbers for the state $j$. The Wigner-Boltzmann equation is now obtained by adding a collision term to the Wigner equation (25) modeling the interaction of the electron with some background. A physically meaningful collision operator which can be used in a reasonable computational context has yet to be developed. There are many derivations of collision operators 
modeling the interaction of electrons with acoustic phonons or systems of coupled oscillators via second quantization approaches [7-9]. Unfortunately, none of them leads to a form which is computationally tractable and, at the same time, is usable in the context of fluid approximations. We will therefore employ a phenomenological collision operator which uses only a minimal amount of information about the actual underlying physical processes. This information consists of the integral invariants, or more precisely, of the balance equations for the moments of the collision operator describing how it affects the balance of particle number, momentum, and energy. We assume that this balance is given by the relaxation terms in Eqs. (1) - (3) and the collision operator of choice is a BGK operator. Thus we consider the Wigner-Boltzmann equation

$$
\partial_{t} F+\frac{1}{m} \nabla_{x} \cdot(p F)-\Theta\left[V+V^{B}\right] F+Q(F)=0,
$$

where the collision operator $Q$ is given by

$$
Q(F)=\frac{1}{\gamma}[F-\Omega(\omega(F), p)], \quad \omega=\left(\omega_{1}, \omega_{2}\right),
$$

with $\gamma$ the relaxation time and $\Omega(\omega, p)$ the desired equilibrium distribution function parametrized by its zero and second order moments:

$$
\int \kappa(p) \Omega(\omega, p) d p=\omega, \quad \kappa:=\left(1,|p|^{2}\right) .
$$

We will in general assume that the equilibrium distribution function $\Omega$ is an even function of each component of the momentum vector $p$, implying that all the odd moments of $\Omega$ vanish and that equilibrium corresponds to a state of zero flux. Computing the moments of the BGK collision operator $Q$ using Eq. (30) gives

$$
\begin{aligned}
M^{0}(Q) & =\frac{1}{\gamma}\left[M^{0}(F)-\omega_{1}\right], M^{1}(Q)=\frac{1}{\gamma} M^{1}(F), \\
\operatorname{trM}^{2}(Q) & =\frac{1}{\gamma}\left[\operatorname{tr} M^{2}(F)-\omega_{2}\right],
\end{aligned}
$$

If we define the moments of the Wigner function $F$ in the usual way via mean velocity and variance as

$$
\begin{gathered}
n:=M^{0}(F), \quad m n u:=M^{1}(F), \\
m\left(w+m n|u|^{2}\right)=t r M^{2}(F),
\end{gathered}
$$

and match the moments of the collision operator with the collision terms in the QHD equations (1) -(3) we obtain

$$
\begin{aligned}
\frac{1}{\gamma}\left(M^{0}(F)-\omega_{1}\right)=0, \quad \frac{1}{\gamma} M^{1}(F) & =\frac{1}{\tau_{p}} m n u \\
& =\frac{1}{\tau_{p}} M^{1}(F), \\
\frac{1}{\gamma}\left[t r M^{2}(F)-\omega_{2}\right] & =\frac{m}{\tau_{w}}\left[w+m n|u|^{2}-3 n T_{0}\right] \\
& =\frac{1}{\tau_{w}}\left(t r M^{2}(F)-3 m T_{0} M^{0}(F)\right),
\end{aligned}
$$

which implies

$$
\begin{aligned}
& \omega_{1}=M^{0}(F), \quad \gamma=\tau_{p}, \\
& \omega_{2}=\left(1-\frac{\tau_{p}}{\tau_{w}}\right) \operatorname{trM}^{2}(F)+\frac{\tau_{p}}{\tau_{w}} 3 m T_{0} M^{0}(F) .
\end{aligned}
$$

Equation (35) gives $\omega=\omega(F)$ as a function of the moments of $F$.

We start by bringing the Wigner-Boltzmann equation (28) into an appropriately scaled and dimensionless form. We scale the spatial variable $x$ by the spatial scale $L$ of the system under consideration (i.e., the device length or the size of the simulation domain), and the momentum vector $p$ by the scale given by the classical thermal equilibrium Maxwell distribution $\bar{p}=\sqrt{m T_{0}}$. We consider the system on the time scale given by the energy relaxation time $\tau_{w}$ in Eq. (3). We scale the potentials $V^{M}$ and $V^{B}$ by

$$
V^{M}(x)=\bar{V}_{M} V_{s}^{M}\left(\frac{x}{L}\right), \quad V^{B}(x)=\bar{V}_{B} V_{s}^{B}\left(\frac{x}{L}\right)
$$


where we denote scaled quantities with a subscript $s$ and $\bar{V}_{M}$ and $\bar{V}_{B}$ are the average typical size of the mean field potential and the barrier height. Scaling the Wigner function $F$ and its moments appropriately gives

$$
\begin{gathered}
F(x, p, t)=\left(\sqrt{m T_{0}} L\right)^{-3} F_{s}\left(x_{s}, p_{s}, t_{s}\right), \\
n(x, t)=L^{-3} n_{s}\left(x_{s}, t_{s}\right), \\
u(x, t)=\sqrt{\frac{T_{0}}{m}} u_{s}\left(x_{s}, t_{s}\right), \\
w(x, t)=\frac{T_{0}}{L^{3}} w_{s}\left(x_{s}, t_{s}\right), \\
P(x, t)=\frac{T_{0}}{L^{3}} P_{s}\left(x_{s}, t_{s}\right), \\
q(x, t)=\frac{\left(m T_{0}\right)^{3 / 2}}{L^{3} m^{2}} q_{s}\left(x_{s}, t_{s}\right),
\end{gathered}
$$

where $x=L x_{s}, p=\sqrt{m T_{0}} p_{s}$, and $t=\tau_{w} t_{s}$. The scaled particle density, scaled velocity, and scaled internal energy density are now related to the moments of the scaled Wigner function via

$$
\begin{array}{r}
n_{s}:=M^{0}\left(F_{s}\right), \quad n_{s} u_{s}:=M^{1}\left(F_{s}\right), \\
w_{s}=\operatorname{trM}^{2}\left(F_{s}^{\prime}\right), \\
P_{s}=M^{2}\left(F_{s}^{\prime}\right), \quad q_{s}=M^{3}\left(F_{s}^{\prime}\right), \\
F_{s}^{\prime}\left(x_{s}, p_{s}, t_{s}\right)=F_{s}\left(x_{s}, p_{s}+u_{s}, t_{s}\right) .
\end{array}
$$

In the same way, we scale the equilibrium distribution function $\Omega$ by

$$
\begin{aligned}
\Omega(\omega, p) & =\left(\sqrt{m T_{0}} L\right)^{-3} \Omega_{s}\left(\omega_{s}, p_{s}\right), \quad \omega_{1}=L^{-3} \omega_{1}^{s}, \\
\omega_{2} & =m T_{0} L^{-3} \omega_{2}^{s},
\end{aligned}
$$

implying that the scaled equilibrium distribution function $\Omega_{s}$ is also described by its moments. The scaled Wigner-Boltzmann equation now reads

$$
\begin{gathered}
\partial_{t_{s}} F_{s}+C \nabla_{x_{s}} \cdot\left(p_{s} F_{s}\right)-\frac{1}{\varepsilon} \Theta\left[V_{s}^{M}\right] F-\Theta\left[V_{s}^{B}\right] F_{s} \\
+\frac{1}{\tau} Q_{s}\left(F_{s}, \tau\right)=0 \\
Q_{s}\left(F_{s}, \tau\right)=F_{s}-\Omega_{s}\left(\alpha\left(F_{s}, \tau\right), p_{s}\right),
\end{gathered}
$$

$$
\begin{aligned}
\Theta[V] & =\frac{i A}{h}\left[V\left(x+\frac{h}{2 i} \nabla_{p}\right)-V\left(x-\frac{h}{2 i} \nabla_{p}\right)\right], \\
h & :=\frac{\hbar}{L \sqrt{m T_{0}}},
\end{aligned}
$$

with $h$ denoting the scaled Planck constant related to the spatial and momentum scales under consideration. The parameters $A, C, \varepsilon$, and $\tau$ are given by

$$
\begin{aligned}
& A=\frac{e \tau_{w} \bar{V}_{B}}{L \sqrt{m T_{0}}}, \quad \varepsilon=\frac{\bar{V}_{B}}{\bar{V}_{M}}, \quad \tau=\frac{\tau_{p}}{\tau_{w}}, \\
& C=\frac{\tau_{w} \sqrt{m T_{0}}}{m L}
\end{aligned}
$$

and the scaled moments $\omega_{s}$ are given in terms of the moments of the scaled Wigner function $F_{s}$ as

$$
\begin{aligned}
\omega_{s} & =\alpha\left(F_{s}, \tau\right), \quad \alpha_{1}\left(F_{s}, \tau\right)=M^{0}\left(F_{s}\right), \\
\alpha_{2}\left(F_{s}, \tau\right) & =(1-\tau) \operatorname{trM}^{2}\left(F_{s}\right)+3 \tau M^{0}\left(F_{s}\right) .
\end{aligned}
$$

Note that the collision operator $Q$ depends explicitly on the parameter $\tau$ since the parameter $\omega$ does. We will consider the following situation: The barrier height (and the resulting energy scale) is commensurate with the energy relaxation time $\tau_{w}$. Thus $A$ and $C$ are $O(1)$. We will also assume that momentum relaxation happens much faster than energy relaxation: $\tau_{p} \ll \tau_{w}, \tau \ll 1$. Moreover, we will assume that the dynamics induced by the external potential $V^{M}$ is somewhat larger than the influence of the barriers: $\varepsilon \ll 1$. This is a relatively weak effect compared to the dominance of the collisions, and we will assume $\tau \ll \varepsilon$. Henceforth we will drop the subscript $s$ for the scaled variables.

We will now express the shifted Wigner function $F^{\prime}$ in terms of the moments of $F$ via a ChapmanEnskog type of expansion. The result of this procedure is the following

Proposition 1 If we define the first moments of the Wigner function $F$ by $n:=M^{0} F$ and $n u:=M^{1} F$ and the centered Wigner function by $F^{\prime}(x, p, t)=$ 
$F(x, p+u, t)$, then $F^{\prime}$ is given asymptotically in terms of $n, u$, and its second moment $w=t r M^{2} F^{\prime}$ as

$$
\begin{aligned}
F^{\prime}(x, p, t)= & \Omega(n, w, p)+u \cdot \nabla_{p} \Omega(n, w, p) \\
& -\Lambda[u] \Omega(n, w, p) \\
& -C \tau\left\{\Lambda\left[\frac{1}{n} \nabla_{x} \cdot M^{2} \Omega\right] \Omega(n, w, p)\right. \\
& \left.\quad+\nabla_{x} \cdot(p \Omega(n, w, p))\right\} \\
& +O\left(\frac{\tau^{2}}{\varepsilon^{2}}\right)
\end{aligned}
$$

with the operator $\Lambda$ given by

$$
\Lambda[U] f:=\frac{1}{2} \int_{-1}^{1} d s\left[U\left(x+\frac{s h}{2 i} \nabla_{p}\right) \cdot \nabla_{p} f\right] .
$$

We will delay the proof of Proposition 1, the actual expansion procedure, to the end of this section, and will first compute the resulting closure conditions for the QHD equations. To this end, we first need to compute the moments of the operator $\Lambda$ in Eq. (49). A straightforward calculation shows

$$
\begin{aligned}
& M^{0}(\Lambda[U] f)=0, \quad M^{1}(\Lambda[U] f)=-U M^{0}(f) \\
& M^{2}(\Lambda[U] f)=-U\left(M^{1}(f)\right)^{T}-M^{1}(f) U^{T} \\
& M^{3}(\Lambda[U] f)=-2 M^{2}(f) U-\left(\operatorname{trM}^{2}(f)\right) U \\
&+\frac{h^{2}}{4} M^{0}(f) \Delta_{x} U
\end{aligned}
$$

(Keep in mind that $M^{1}$ and $M^{3}$ are defined as vectors and $M^{2}$ is a matrix). Computing the moments of $F^{\prime}$ asymptotically from Eq. (48), i.e., dropping the $O\left(\tau^{2} / \lambda^{2}\right)$ term, we obtain

$$
\begin{aligned}
M^{0} F^{\prime}=n, \quad P=M^{2} F^{\prime} & =M^{2} \Omega(n, w), \\
t r M^{2} F^{\prime} & =w .
\end{aligned}
$$

Computing the third moment of each of the terms in Eq. (48) gives

$$
M^{3}\left[u \cdot \nabla_{p} \Omega(n, w, p)\right]=-w u-2\left(M^{2} \Omega(n, w)\right) u,
$$

$$
\begin{gathered}
M^{3}(\Lambda[u] \Omega(n, w, p))=-2\left(M^{2} \Omega(n, w)\right) u-w u \\
+\frac{h^{2} n}{4} \Delta_{x} u \\
M^{3}\left\{\Lambda\left[\frac{1}{n} \nabla_{x} \cdot M^{2} \Omega\right] \Omega(n, w, p)+\nabla_{x} \cdot(p \Omega(n, w, p))\right\} \\
=-\frac{2}{n}\left(M^{2} \Omega\right)\left(\nabla_{x} \cdot M^{2} \Omega\right)-\frac{w}{n} \nabla_{x} \cdot M^{2} \Omega \\
+\frac{h^{2} n}{4} \Delta_{x}\left(\frac{1}{n} \nabla_{x} \cdot M^{2} \Omega\right)+\nabla_{x} \cdot M^{4} \Omega .
\end{gathered}
$$

Using the fact that $M^{2} \Omega=P$, we obtain the heat flux by building the third moment $M^{3}$ of Eq. (48) using Eqs. (54)-(56):

$$
\begin{aligned}
q=-\frac{h^{2} n}{4} \Delta_{x} u+C \tau[ & \frac{2}{n} P\left(\nabla_{x} \cdot P\right)+\frac{w}{n} \nabla_{x} \cdot P \\
& -\frac{h^{2} n}{4} \Delta_{x}\left(\frac{1}{n} \nabla_{x} \cdot P\right) \\
& \left.-\nabla_{x} \cdot M^{4} \Omega(n, w)\right] .
\end{aligned}
$$

Thus, given an equilibrium distribution function $\Omega$, the stress tensor and the heat flux are expressed in terms of the primary variables $n, u$, and $w$. In unscaled variables the closure conditions for the QHD system now read

$$
P=\frac{1}{m} M^{2} \Omega(n, m w),
$$

$$
\begin{aligned}
q= & -\frac{\hbar^{2} n}{4 m} \Delta_{x} u+\frac{2 \tau_{p}}{m n} P\left(\nabla_{x} \cdot P\right)+\frac{\tau_{p} w}{m n} \nabla_{x} \cdot P \\
& -\frac{\hbar^{2} \tau_{p} n}{4 m^{2}} \Delta_{x}\left(\frac{1}{n} \nabla_{x} \cdot P\right)-\frac{\tau_{p}}{m^{3}} \nabla_{x} \cdot M^{4} \Omega(n, m w) .
\end{aligned}
$$

Since the closure now contains additional derivatives in the spatial direction, i.e., diffusive terms, one immediate question is whether these terms have the right sign for a well posed problem. The answer to this question will of course depend on 
the form of the equilibrium distribution function $\Omega$ used in the BGK collision operator. We simplify the expression for the heat flux by splitting $\Omega$ into its classical and quantum components. Whatever approximation we use, we can assume that $\Omega$ will reduce to a classical Maxwellian in the classical limit $\hbar \rightarrow 0$. The classical Maxwellian, parametrized by its moments, is of the form

$$
\Omega^{C}(n, w, p)=n\left(\frac{3 n}{2 \pi w}\right)^{3 / 2} \exp \left[-\frac{3 n|p|^{2}}{2 w}\right]
$$

and satisfies $M^{2} \Omega^{C}(n, w)=(w / 3) I \quad$ and $M^{4} \Omega^{C}(n, w)=\left(5 w^{2} / 9 n\right) I$. If we set $\Omega=\Omega^{C}+\hbar^{2} \Omega^{Q}$ and, accordingly, $P=P^{C}+\hbar^{2} P^{Q}$ and $q=q^{C}+{ }^{-} h^{2} q^{Q}$, we obtain

$$
\begin{gathered}
P^{C}=\frac{w}{3} I, \quad P^{Q}=\frac{1}{m} M^{2} \Omega^{Q}(n, m w), \\
q^{C}=-\frac{5 \tau_{p} w}{9 m} \nabla_{x}\left(\frac{w}{n}\right), \\
q^{Q}=-\frac{n}{4 m} \Delta_{x} u+\frac{\tau_{p}}{m n}\left[\frac{5 w}{3} I+2 \hbar^{2} P^{Q}\right]\left(\nabla_{x} \cdot P^{Q}\right) \\
+\frac{\tau_{p}}{m n} P^{Q} \nabla_{x} \frac{w}{3} \\
-\frac{\tau_{p} n}{4 m^{2}} \Delta_{x}\left[\frac{1}{n} \nabla_{x} \cdot\left(\frac{w}{3} I+\hbar^{2} P^{Q}\right)\right] .
\end{gathered}
$$

This shows that the classical part of the heat flux is given by the usual heat conduction term corresponding to Fourier's law. For regimes which are close to the classical case, i.e., for space and time scales for which $h$ is small, one will drop the $O\left(\tau_{p}\right)$ terms in $q^{C}$. In this case the closed QHD system is of the form

$$
\begin{gathered}
\partial_{t} n+\nabla_{x} \cdot(n u)=0, \\
\partial_{t}(m n u)+\nabla_{x} \cdot\left[m n u u^{T}+\hbar^{2} P^{Q}\right]+\nabla_{x}\left(\frac{w}{3}\right) \\
+e n \nabla_{x} V^{\text {tot }}+\frac{m n u}{\tau_{p}}=0,
\end{gathered}
$$

$$
\begin{aligned}
\partial_{t}\left(w+m|u|^{2} n\right)+\nabla_{x} \cdot[ & \frac{5 w}{3} u+m u|u|^{2} n \\
& -\frac{5 \tau_{p} w}{9 m} \nabla_{x}\left(\frac{w}{n}\right) \\
& \left.-\frac{\hbar^{2} n}{4 m} \Delta_{x} u+2 \hbar^{2} P^{Q} u\right] \\
+2 e n\left(\nabla_{x} V^{\mathrm{tot}}\right) \cdot u & +\frac{\left(w-3 n T_{0}\right)+m|u|^{2} n}{\tau_{w}}=0 .
\end{aligned}
$$

The closure is now completely determined by the quantum part of the second moment matrix.

Proof of Proposition 1 Before we start the expansion procedure, we will perform a transformation of the Wigner-Boltzmann equation which will simplify the algebra. We will split the Wigner function $F$ into its even and odd parts with respect to $p$, noting that the equilibrium distribution function $\Omega$ is by assumption an even function of $p$, and that the field operator $\Theta$ maps even functions of $p$ into odd functions and vice versa. A closer inspection of Eq. (43) indicates that the odd part of $F$ will be small, namely $O(\tau / \varepsilon)$. We therefore set

$$
F=f+\frac{\tau}{\varepsilon} g
$$

$$
\begin{aligned}
& f(x, p, t)=\frac{1}{2}[F(x, p, t)+F(x,-p, t)], \\
& g(x, p, t)=\frac{\varepsilon}{2 \tau}[F(x, p, t)-F(x,-p, t)] .
\end{aligned}
$$

Furthermore, we will write the symbol of the pseudo-differential operator $\Theta$ as a function of the electric field rather than as a function of the potential. We use the identity

$$
V(x+z)-V(x-z)=\int_{-1}^{1} \nabla_{x} V(x+s z) \cdot z d s,
$$


to write

$$
\begin{aligned}
\Theta[V] & =\frac{i A}{h}\left[V\left(x+\frac{h}{2 i} \nabla_{p}\right)-V\left(x-\frac{h}{2 i} \nabla_{p}\right)\right] \\
& =A \Lambda\left[\nabla_{x} V\right] \\
\Lambda[U] & :=\frac{1}{2} \int_{-1}^{1} d s\left[U\left(x+\frac{s h}{2 i} \nabla_{p}\right) \cdot \nabla_{p}\right] .
\end{aligned}
$$

Splitting the Wigner-Boltzmann equation into its even and odd parts gives

$$
\begin{gathered}
\tau \partial_{t} f+\frac{\tau^{2}}{\varepsilon} C \nabla_{x} \cdot(p g)-\frac{\tau^{2}}{\varepsilon^{2}} A \Lambda\left[\nabla_{x} V^{M}\right] g \\
-\frac{\tau^{2}}{\varepsilon} A \Lambda\left[\nabla_{x} V^{B}\right] g+Q(f, \tau)=0 \\
\tau \partial_{t} g+\varepsilon C \nabla_{x} \cdot(p f)-A \Lambda\left[\nabla_{x} V^{M}\right] f \\
-\varepsilon A \Lambda\left[\nabla_{x} V^{B}\right] f+g=0
\end{gathered}
$$

The even part of the collision operator $Q$ is then defined by

$$
\begin{gathered}
Q(f, \tau)=f-\Omega(\alpha(f, \tau), p), \\
\alpha=\left(\alpha_{1}, \alpha_{2}\right), \quad \alpha_{1}(f, \tau)=M^{0}(f), \\
\alpha_{2}(f, \tau)=(1-\tau) t r M^{2}(f)+3 \tau M^{0}(f) .
\end{gathered}
$$

We will derive a Chapman-Enskog type asymptotic solution to the scaled Wigner-Boltzmann equation (43). That is we will derive an asymptotic expression of the form

$$
\begin{aligned}
& f(x, p, t, \tau, \varepsilon) \approx \phi\left(M^{0} f, M^{1} g, t r M^{2} f, p, \tau, \varepsilon\right), \\
& g(x, p, t, \tau, \varepsilon) \approx \psi\left(M^{0} f, M^{1} g, t r M^{2} f, p, \tau, \varepsilon\right),
\end{aligned}
$$

where the shape functions $\phi$ and $\psi$ only depend on space and time through the moments of $f$ and $g$. We start by setting $\tau=\varepsilon=0$ in Eqs. (71), (72) and obtain for the zero order term

$$
\begin{aligned}
0= & Q\left(f_{0}, 0\right)=f_{0}-\Omega\left(\alpha\left(f_{0}, 0\right), p\right), \\
& -A \Lambda\left[\nabla_{x} V^{M}\right] f_{0}+g_{0}=0,
\end{aligned}
$$

which implies

$$
f_{0}=\Omega\left(\omega^{0}, p\right), \quad g_{0}=A \Lambda\left[\nabla_{x} V^{M}\right] \Omega\left(\omega^{0}, p\right),
$$

for a general arbitrary parameter $\omega^{0}$. In order to compute the zero order shape functions $\phi_{0}$ and $\psi_{0}$ we have to write $f_{0}$ and $g_{0}$ and therefore $\omega^{0}$ in terms of the moments $n_{0}:=M^{0} f_{0}, J_{0}:=M^{1} g_{0}$, and $w_{0}:=\operatorname{tr} M^{2} f_{0}$. At this point we have to consider the moments of the operator $\Lambda$. This gives

$$
\omega_{1}^{0}=n_{0}, \omega_{2}^{0}=w_{0}, J_{0}=M^{1}\left(g_{0}\right)=-A \nabla_{x} V^{M} n_{0} .
$$

Thus, we can in zero order write $f_{0}$ and $g_{0}$ in terms of their moments as

$$
\begin{gathered}
f_{0}=\phi_{0}\left(M^{0} f, M^{2} f, p\right), \\
g_{0}=\psi_{0}\left(M^{0} f, M^{1} g, M^{2} f, p\right), \\
\phi_{0}(n, w, p)=\Omega(n, w, p), \\
\psi_{0}(n, J, w, p)=-\Lambda\left[\frac{J}{n}\right] \Omega(n, w, p) .
\end{gathered}
$$

Since we have assumed that $\tau \ll \varepsilon$, the next terms in the expansion of $f$ and $g$ have to be of the form $\tau f_{1}$ and $\varepsilon g_{1}$, where $g_{1}$ satisfies

$$
\begin{gathered}
C \nabla_{x} \cdot\left(p f_{0}\right)-A \Lambda\left[\nabla_{x} V^{B}\right] f_{0}+g_{1}=0 \\
g_{1}=A \Lambda\left[\nabla_{x} V^{B}\right] \Omega\left(n_{0}, w_{0}, p\right) \\
-C \nabla_{x} \cdot\left(p \Omega\left(n_{0}, w_{0}, p\right)\right)
\end{gathered}
$$

The term $f_{1}$ is actually not relevant for our development. Computing the first moment of $g_{1}$ using Eqs. $(50)-(52)$ gives

$$
\begin{aligned}
J_{1}:= & M^{1} g_{1}=-A n_{0} \nabla_{x} V^{B}-C \nabla_{x} \cdot M^{2} \Omega\left(n_{0}, w_{0}\right) \\
= & -\nabla_{x} \cdot\left[A n V^{B} I+C M^{2} \Omega\left(n_{0}, w_{0}\right)\right] \\
& +A V^{B} \nabla_{x} n_{0},
\end{aligned}
$$

where we have written $J_{1}$ in divergence form since the barrier potential $V^{B}$ is a discontinuous function. This shows that in order for this expansion procedure to make sense, the equilibrium distribution function $\Omega$ has to be of a form such that the term inside the divergence in Eq. (83) remains continuous. We assume for the moment that this is 
the case. We then have to compute the next term in the expansion of the shape function $\psi$ :

$$
\begin{aligned}
& \psi\left(M^{0} f, M^{1} g, t r M^{2} f, p, \tau, \varepsilon\right) \\
& =\psi_{0}\left(M^{0} f, M^{1} g, t r M^{2} f, p\right) \\
& \quad+\varepsilon \psi_{1}\left(M^{0} f, M^{1} g, t r M^{2} f, p\right)+O(\tau),
\end{aligned}
$$

or

$$
\begin{aligned}
g_{0}+\varepsilon g_{1}= & \psi_{0}\left(n_{0}+\tau n_{1}, J_{0}+\varepsilon J_{1}, w_{0}+\tau w_{1}, p\right) \\
& +\varepsilon \psi_{1}\left(n_{0}+\tau n_{1}, J_{0}+\varepsilon J_{1}, w_{0}+\tau w_{1}, p\right) \\
& +O(\tau),
\end{aligned}
$$

which gives for $\tau \rightarrow 0, \varepsilon \rightarrow 0$, and $(\tau / \varepsilon) \rightarrow 0$

$$
g_{1}=\frac{\partial \psi_{0}}{\partial J}\left(n_{0}, J_{0}, w_{0}, p\right) J_{1}+\psi_{1}\left(n_{0}, J_{0}, w_{0}, p\right) .
$$

We note that the shape function $\psi_{0}$ depends linearly on the flux $J$, and therefore the Frechet derivative of $\psi_{1}$ in Eq. (86) is given by

$$
\frac{\partial \psi_{0}}{\partial J}\left(n_{0}, J_{0}, w_{0}, p\right) J_{1}=-\Lambda\left[\frac{J_{1}}{n_{0}}\right] \Omega\left(n_{0}, w_{0}, p\right) .
$$

Bringing this term on the other side we obtain the functional form of $\psi_{1}$ as

$$
\begin{aligned}
\psi_{1}\left(n, w, p, V^{B}\right)= & A \Lambda\left[\nabla_{x} V^{B}\right] \Omega(n, w, p) \\
& -C \nabla_{x} \cdot(p \Omega(n, w, p)) \\
& -\Lambda\left[\frac{Z}{n}\right] \Omega(n, w, p), \\
Z:=A n \nabla_{x} V^{B} & +C \nabla_{x} \cdot M^{2} \Omega(n, w) .
\end{aligned}
$$

Combining the terms involving the operator $\Lambda$ gives

$$
\begin{aligned}
\psi_{1}\left(n, w, p, V^{B}\right)= & -C \Lambda\left[\frac{1}{n} \nabla_{x} \cdot M^{2} \Omega\right] \Omega(n, w, p) \\
& -C \nabla_{x} \cdot(p \Omega(n, w, p)) .
\end{aligned}
$$

Thus, in summary, we have expanded the Wigner function in terms of its moments as

$$
\begin{aligned}
F= & f+\frac{\tau}{\varepsilon} g=\phi_{0}(n, w, p)+\frac{\tau}{\varepsilon} \psi_{0}(n, J, w, p) \\
& +\tau \psi_{1}\left(n, w, p, V^{B}\right)+O\left(\frac{\tau^{2}}{\varepsilon^{2}}\right)
\end{aligned}
$$

$$
\begin{aligned}
& n=M^{0} f=M^{0} F, \quad J=M^{1} g=\frac{\varepsilon}{\tau} M^{1} F, \\
& w=t r M^{2} f=t r M^{2} F .
\end{aligned}
$$

Note that by virtue of the construction, $\phi_{0}$ is an even function of $p$ with vanishing moments of odd order, and $\psi_{0}$ and $\psi_{1}$ are odd functions with vanishing moments of even order. To evaluate the stress tensor and the heat flux we have to compute the shifted Wigner function $F^{\prime}(x, p, t)=F(x$, $p+u, t)$ where $u$ is the mean momentum vector given by $M^{1} F=u M^{0} F$. From Eq. (92) we see that $u=(\tau / \varepsilon n) J$. Thus $F^{\prime}$ is given by

$$
\begin{aligned}
F^{\prime}(x, p, t)= & \phi_{0}\left(n, w, p+\frac{\tau}{\varepsilon n} J\right) \\
& +\frac{\tau}{\varepsilon} \psi_{0}\left(n, J, w, p+\frac{\tau}{\varepsilon n} J\right) \\
& +\tau \psi_{1}\left(n, w, p+\frac{\tau}{\varepsilon n} J, V^{B}\right)+O\left(\frac{\tau^{2}}{\varepsilon^{2}}\right) \\
= & \phi_{0}(n, w, p)+\frac{\tau}{\varepsilon n} J \cdot \nabla_{p} \phi_{0}(n, w, p) \\
& +\frac{\tau}{\varepsilon} \psi_{0}(n, J, w, p)+\tau \psi_{1}\left(n, w, p, V^{B}\right) \\
& +O\left(\frac{\tau^{2}}{\varepsilon^{2}}\right) .
\end{aligned}
$$

Computing the second moment $w=\operatorname{tr} M^{2} F^{\prime}$ of the shifted Wigner function $F^{\prime}$ from Eq. (93) gives $3 n T=w$, because $\nabla_{p} \phi_{0}, \psi_{0}$, and $\psi_{1}$ are all odd functions of $p$. Thus the shifted Wigner function satisfies

$$
\begin{aligned}
F^{\prime}(x, p, t)= & \Omega(n, w, p)+\frac{\tau}{\varepsilon n} J \cdot \nabla_{p} \Omega(n, w, p) \\
& -\frac{\tau}{\varepsilon} \Lambda\left[\frac{J}{n}\right] \Omega(n, w, p) \\
& -\tau C \Lambda\left[\frac{1}{n} \nabla_{x} \cdot M^{2} \Omega\right] \Omega(n, w, p) \\
& -\tau C \nabla_{x} \cdot(p \Omega(n, w, p))+O\left(\frac{\tau^{2}}{\varepsilon^{2}}\right) .
\end{aligned}
$$

Replacing $J$ by $(\varepsilon / \tau) n u$ gives Eq. (48). 


\section{ASYMPTOTIC APPROXIMATIONS TO THERMAL EQUILIBRIUM}

In this section we derive asymptotic approximations to the thermal equilibrium distribution function corresponding to Boltzmann distributed states. The goal here is to find a sufficiently simple expression for the equilibrium distribution function $\Omega$ in Section 2, which is parametrized by its moments in the momentum direction. In general, the density matrix operator $\rho$ corresponding to a system of eigenstates of the Hamiltonian $H=\left(-\hbar^{2} / 2 m\right) \Delta_{r}+$ $V$ is given by $\rho=g(H)$ or, more precisely by

$$
\begin{aligned}
(\rho f)(r) & =\sum_{n} g\left(\lambda_{n}\right) c_{n} \psi_{n}(r), \\
c_{n} & :=\int f(s) \psi_{n}(s) d s,
\end{aligned}
$$

where the $\psi_{n}$ 's are the normalized eigenfunctions of the Hamiltonian $H$ satisfying

$$
\begin{aligned}
& \lambda_{n} \psi_{n}=H \psi_{n}=\frac{-\hbar^{2}}{2 m} \Delta_{r} \psi_{n}+V \psi_{n}, \\
& \int\left|\psi_{n}(r)\right|^{2} d r=1 .
\end{aligned}
$$

Rewriting Eq. (95), and using the same symbol for the operator $\rho$ as for its integral kernel, gives

$$
\begin{aligned}
(\rho f)(r) & =\int \rho(r, s) f(s) d s, \\
\rho(r, s) & =\sum_{n} g\left(\lambda_{n}\right) \psi_{n}(r) \psi_{n}(s) .
\end{aligned}
$$

The goal of this section is to derive approximate formulae for the integral kernel $\rho$ in the case of Boltzmann distributed states when the distribution function $g$ is given by $g(\lambda)=\exp [-(\lambda / \tau)]$. The approach for this approximation will be via the Bloch equation [6]. A direct calculation shows that the integral kernel $\rho$ of the operator $e^{-\beta H}$, as defined in Eq. (97), satisfies the initial value problem

$$
\begin{array}{r}
\partial_{\beta} \rho=\frac{\hbar^{2}}{4 m}\left[\Delta_{r}+\Delta_{s}\right] \rho-\frac{e}{2}(V(r)+V(s)) \rho, \\
\rho(r, s, \beta=0)=\delta(r-s) .
\end{array}
$$

The initial condition is obtained from the fact that for $\beta=0$ the operator $\rho$ equals the identity operator, and the integral kernel $\rho$ in Eq. (97) can be obtained from solving the initial value problem (98) and evaluating the solution at $\beta=$ $1 / T$. Since we will be particularly interested in the moments of the Wigner function [10] corresponding to the density matrix operator $\rho$, it is convenient to introduce the Wigner transform. We define the Wigner transform of the equilibrium density matrix by

$$
\Omega(x, p, \beta)=\int \rho\left(x-\frac{\hbar}{2} \eta, x+\frac{\hbar}{2} \eta, \beta\right) \exp (i \eta \cdot p) d \eta .
$$

The equilibrium Wigner function $\Omega$ satisfies the transformed initial value problem

$$
\begin{gathered}
\partial_{\beta} \Omega=\frac{\hbar^{2}}{8 m} \Delta_{x} \Omega-\frac{1}{2 m}|p|^{2} \Omega \\
-\frac{e}{2}\left[V\left(x+\frac{\hbar}{2 i} \nabla_{p}\right)+V\left(x-\frac{\hbar}{2 i} \nabla_{p}\right)\right] \Omega \\
\Omega(x, p, \beta=0)=\hbar^{-3}
\end{gathered}
$$

It is the Bloch equation in the form (100) from which asymptotic approximations are most easily derived. In order to do so we first introduce scales bringing Eq. (100) into a dimensionless form. The spatial scales will be given by the variation of the potential. The potential $V$ of the Hamiltonian in Eq. (96) consists of two parts. We have already seen in the previous section that the barriers should be included in the equilibrium solution. Thus we set $V=V^{B}+U$ in Eqs. (96) and (100) where $V^{B}$ is the piecewise constant barrier potential and $U$ is a potential which scales the amplitude of the equilibrium distribution function $\Omega$. We denote the spatial scales and amplitudes of these potentials by $L_{U}, L_{B}, \tilde{U}$, and $\tilde{V}_{B}$, and define the correspondingly scaled potentials by

$$
U(x)=\tilde{U} U_{s}\left(\frac{x}{L_{U}}\right), \quad V^{B}(x)=\tilde{V}_{B} V_{s}^{B}\left(\frac{x}{L_{B}}\right) .
$$


We scale $\beta$ by $1 / T_{0}$ and $p$ by $\sqrt{m T_{0}}$. We therefore scale the equilibrium Wigner function by $\Omega(x, p, \beta)=\hbar^{-3} \Omega_{s}\left(\left(x / L_{U}\right),\left(p / \sqrt{m T_{0}}\right), T_{0} \beta\right)$ giving the scaled Bloch equation

$$
\begin{array}{cr}
\partial_{\beta} \Omega_{s}(x, p, \beta)=\frac{\lambda^{2} h^{2}}{8} \Delta_{x} \Omega_{s}-\frac{1}{2}|p|^{2} \Omega_{s} & (102) \\
-\Theta\left[U_{s}\right] \Omega_{s}-\varepsilon \Theta\left[V_{s}^{B}\right] \Omega_{s}, & (103) \\
\Omega_{s}(x, p, \beta=0)=1, & (104) \\
\Theta\left[U_{s}\right]=\frac{A}{2}\left[U_{s}\left(x+\frac{h \lambda}{2 i} \nabla_{p}\right)+U_{s}\left(x-\frac{h \lambda}{2 i} \nabla_{p}\right)\right], \\
\Theta\left[V_{s}^{B}\right]=\frac{A}{2}\left[V_{s}^{B}\left(\frac{x}{\lambda}+\frac{h}{2 i} \nabla_{p}\right)+V_{s}^{B}\left(\frac{x}{\lambda}-\frac{h}{2 i} \nabla_{p}\right)\right], \\
h=\frac{\hbar}{L_{B} \sqrt{m T_{0}}}, A=\frac{e \tilde{U}}{T_{0}}, \varepsilon=\frac{\tilde{V}_{B}}{\tilde{U}}, \lambda=\frac{L_{B}}{L_{U}} . & (106)
\end{array}
$$

Thus $h$ now denotes the Planck constant in units corresponding to the ambient temperature $T_{0}$ and the spatial scale of the barrier potential, and $A$ is the size of the mean field potential energy in units corresponding to the ambient temperature. The scaled equilibrium distribution function is now obtained by evaluating $\Omega_{s}$ at $\beta=T_{0} / T$. The basic assumption of the following development is that the potential $U$ varies slowly in space compared to the barrier potential $V^{B}$, and that the barrier potential is small compared to $U$, i.e., $\varepsilon \ll 1$, $\lambda \ll 1$. We will assume a situation where the scaled Planck constant is $O(1)$ on the length scale of the barriers, implying that quantum effects are mainly important close to the barrier. That is we will derive asymptotic approximations for the regime $A=O(1), h=O(1), \varepsilon \ll 1, \lambda \ll 1$. Henceforth we will drop the subscript $s$.

Moreover, instead of deriving a straightforward expansion of the equilibrium Wigner function $\Omega$ in powers of the parameters $\varepsilon$ and $\lambda$, we will, as in the previous section, derive a Chapman-Enskog type expansion where the terms in the expansion of $\Omega$ depend on the spatial variable $x$ only through its zero and second order moments, which we denote by the vector $\omega=\left(\omega_{1}, \omega_{2}\right)=\left(M^{0} \Omega, \operatorname{tr} M^{2} \Omega\right)$, and through the barrier potential $V^{B}$. Thus we expand the solution $\Omega$ of Eq. (102) in the form

$$
\begin{aligned}
& \Omega(x, p, \beta, \varepsilon, \lambda) \approx \sum_{k=0}^{\infty} \Omega_{k}(x, p, \beta, \lambda) \varepsilon^{k}, \\
& \Omega_{k}(x, p, \beta, \lambda) \approx \sum_{j=0}^{\infty} \Omega_{k j}(x, p, \beta) \lambda^{2 j},
\end{aligned}
$$

and use this result to write the equilibrium distribution function as

$$
\begin{aligned}
& \Omega(x, p, \beta, \varepsilon, \lambda) \approx \sum_{k=0}^{\infty} \phi_{k}\left(\omega, V^{B}, p, \beta, \lambda\right) \varepsilon^{k} \\
& \phi_{k}\left(\omega, V_{B}, p, \beta, \lambda\right) \approx \sum_{j=0}^{\infty} \phi_{k j}\left(\omega, V^{B}, p, \beta\right) \lambda^{2 j} .
\end{aligned}
$$

We note that formally replacing $\lambda$ by $-\lambda$ in Eq. (102) does not change the equation and hence we can expand in only even powers of $\lambda$. The Chapman-Enskog expansion of the Bloch equation (102) yields the following

Proposition 2 The equilibrium distribution function $\Omega$ is asymptotically given in terms of its moment vector $\omega=\left(M^{0}\right.$, tr $\left.M^{2}\right) \Omega$ and the barrier potential $V^{B}$ by the expression

$$
\begin{aligned}
\Omega(x, p, \beta)= & \phi_{00}(\omega, p)+\lambda^{2} \phi_{01}(\omega, p)+\varepsilon \phi_{10}\left(\omega, p, V^{B}\right) \\
& +o\left(\lambda^{2}\right)+o(\varepsilon),
\end{aligned}
$$

with the functions $\phi_{00}, \phi_{01}$, and $\phi_{10}$ given by

$$
\begin{aligned}
\phi_{00}(\omega, p)= & \omega_{1}\left(\frac{\theta}{2 \pi}\right)^{3 / 2} \exp \left(-\frac{\theta}{2}|p|^{2}\right), \quad \theta:=\frac{3 \omega_{1}}{\omega_{2}}, \\
\phi_{01}(\omega, p)= & \frac{h^{2}}{24} \omega_{1}\left(\frac{\theta}{2 \pi}\right)^{3 / 2} \exp \left(-\frac{\theta}{2}|p|^{2}\right) \theta^{3} \\
& \sum_{\mu \nu}\left(\partial_{x_{\mu \nu}}^{2} H\right)\left[p_{\mu} p_{\nu}-\frac{\delta_{\mu \nu}}{3}|p|^{2}\right],
\end{aligned}
$$




$$
H:=\frac{1}{\theta}\left[-\ln \omega_{1}-\frac{3}{2} \ln \left(\frac{\theta}{2 \pi}\right)\right],
$$

$$
\begin{aligned}
\phi_{10}\left(\omega, p, V^{B}\right) \\
=\frac{1}{2} A \theta\left(\frac{\theta}{2 \pi}\right)^{3 / 2} \omega_{1} \\
\left\{-2 S\left(\sqrt{\theta} p, \frac{h \sqrt{\theta}}{2 i} \nabla_{y}\right)\right. \\
+\exp \left(-\frac{\theta}{2}|p|^{2}\right) \\
\quad\left[\left(2 \Gamma_{0}+\frac{\theta h^{2}}{4} \Delta_{y} \Gamma_{2}\right)\left(\frac{h \sqrt{\theta}}{2 i} \nabla_{y}\right)\right. \\
\left.\left.\quad-\frac{h^{2} \theta^{2}}{12}|p|^{2} \Delta_{y} \Gamma_{2}\left(\frac{h \sqrt{\theta}}{2 i} \nabla_{y}\right)\right]\right\}\left.V^{B}(y)\right|_{y=x / \lambda},
\end{aligned}
$$

where the dependence of $\phi_{10}$ on the barrier potential $V^{B}$ is of a functional form involving the pseudo-differential operators $S, \Gamma_{0}$, and $\Gamma_{2}$ defined by

$$
\begin{gathered}
S(p, \xi)=\frac{1}{p \cdot \xi} \sinh (p \cdot \xi) \exp \left[-\frac{1}{2}|\xi|^{2}-\frac{1}{2}|p|^{2}\right] \\
\Gamma_{0}(\xi)=\int_{0}^{1} \exp \left[\frac{|\xi|^{2}}{2}\left(\gamma^{2}-1\right)\right] d \gamma, \\
\Gamma_{2}(\xi)=\int_{0}^{1} \gamma^{2} \exp \left[\frac{|\xi|^{2}}{2}\left(\gamma^{2}-1\right)\right] d \gamma
\end{gathered}
$$

The symbols of the operators $\Gamma_{0}$ and $\Gamma_{2}$ are related to the moments of the symbol of the operator $S$ in the momentum direction via

$$
\begin{aligned}
& M^{0} S(\xi)=(2 \pi)^{3 / 2} \Gamma_{0}(\xi) \\
& M^{2} S(\xi)=(2 \pi)^{3 / 2}\left[\Gamma_{0}(\xi) I+\Gamma_{2}(\xi) \xi \xi^{T}\right]
\end{aligned}
$$

We defer the proof of Proposition 2 to the end of this section. For the Chapman-Enskog expansion of the Wigner-Boltzmann equation in Section 2 we will need all the second order moments of this asymptotic solution of the Bloch equation expressed in terms of the moment vector $\omega$; i.e., we will need the full matrix $M^{2} \Omega$ consisting of the integrals $\int p_{j} p_{k} \Omega d p, j, k=1,2,3$ in order to close the system. Since we have used a slightly different scaling of the equilibrium Wigner function $\Omega$ to derive the asymptotic expression (109), we will reverse this scaling before we compute the stress tensor. In unscaled variables the approximation (109) is given by

$$
\begin{aligned}
\Omega= & \phi_{00}+\phi_{01}+\phi_{10}+o\left(\lambda^{2}\right)+o(\varepsilon) \\
\omega= & \left(M^{0}, t r M^{2}\right) \Omega \\
\phi_{00}= & \omega_{1}\left(\frac{\theta}{2 \pi}\right)^{3 / 2} \exp \left(-\frac{\theta}{2}|p|^{2}\right), \quad \theta:=\frac{3 \omega_{1}}{\omega_{2}} \\
\phi_{01}= & \frac{\hbar^{2}}{24} \omega_{1}\left(\frac{\theta}{2 \pi}\right)^{3 / 2} \exp \left(-\frac{\theta}{2}|p|^{2}\right) \theta^{3} \\
& \sum_{\mu \nu}\left(\partial_{x_{\mu \nu}}^{2} H\right)\left[p_{\mu} p_{\nu}-\frac{\delta_{\mu \nu}}{3}|p|^{2}\right] \\
H= & \frac{1}{\theta}\left[-\ln \omega_{1}-\frac{3}{2} \ln \left(\frac{\theta \hbar^{2}}{2 \pi}\right)\right] \\
\phi_{10}= & \frac{m e}{2} \theta\left(\frac{\theta}{2 \pi}\right)^{3 / 2} \omega_{1} \\
& \left\{-2 S\left(\sqrt{\theta} p, \frac{\hbar \sqrt{\theta}}{2 i} \nabla_{x}\right)\right. \\
& +\exp \left(-\frac{\theta}{2}|p|^{2}\right) \\
& \left.\left.-\frac{\hbar^{2} \theta^{2}}{12}|p|^{2} \Delta_{x} \Gamma_{2}\left(\frac{\hbar \sqrt{\theta}}{2 i} \nabla_{x}\right)\right]\right\} \\
V^{B}(x) & \left(2 \Gamma_{0}+\frac{\theta \hbar^{2}}{4} \Delta_{x} \Gamma_{2}\right)\left(\frac{\hbar \sqrt{\theta}}{2 i} \nabla_{x}\right) \\
& -122) \\
&
\end{aligned}
$$

The stress tensor $P=P^{C}+\hbar^{2} P^{Q}$ is now obtained by computing the second order moment matrix $M^{2}$ of each of the above expressions. The second order moments of the shape functions $\phi$ satisfy

$$
\left(M^{2} \phi_{00}\right)_{j k}=\frac{\omega_{2}}{3} \delta_{j k}
$$




$$
\begin{aligned}
& \left(M^{2} \phi_{01}\right)_{j k}=\frac{\hbar^{2}}{12} \omega_{1} \theta\left(\partial_{x_{j k}} H-\frac{1}{3} \delta_{j k} \Delta_{x} H\right), \\
& \theta:=\frac{3 \omega_{1}}{\omega_{2}}, \\
& \left(M^{2} \phi_{10}\right)_{j k}=\theta \omega_{1} \frac{m e \hbar^{2}}{12}\left\{3 \partial_{x_{j k}}-\delta_{j k} \Delta_{x}\right\} \\
& \Gamma_{2}\left(\frac{\hbar \sqrt{\theta}}{2 i} \nabla_{x}\right) V^{B}(x)
\end{aligned}
$$

and $P$ is obtained from evaluating Eqs. (123)(125) at $\omega_{1}=n$ and $\omega_{2}=m w$ and dividing by the mass $m$. Splitting $P^{Q}$ into $P^{Q}=P^{Q 1}+P^{Q^{2}}$, corresponding to the terms $\phi_{01}$ and $\phi_{10}$, gives

$$
\begin{gathered}
P_{j k}^{C}=\frac{w}{3} \delta_{j k}, \\
P_{j k}^{Q 1}=\frac{n^{2}}{4 m w}\left(\partial_{x_{j k}} H-\frac{1}{3} \delta_{j k} \Delta_{x} H\right), \\
H=\frac{w}{3 n}\left[-\ln n+\frac{3}{2} \ln \left(\frac{2 \pi m w}{3 n \hbar^{2}}\right)\right] \\
P_{j k}^{Q 2}=\frac{e n^{2}}{4 m w}\left\{3 \partial_{x_{j k}}-\delta_{j k} \Delta_{x}\right\} \\
\Gamma_{2}\left(\frac{\hbar}{2 i \sqrt{m w / 3 n}} \nabla_{x}\right) V^{B}(x) .
\end{gathered}
$$

Thus, using the expressions in Eq. (126) for the stress tensor $P^{Q}=P^{Q 1}+P^{Q 2}$ in Eq. (61), the quantum hydrodynamic system is closed and a solution can be computed in terms of the primary variables $n, u$, and $w$. From a physical standpoint it is desirable to express the internal energy density $w$ in terms of a temperature $T$. Mathematically, this just corresponds to a simple change of variables once a relation between $w$ and $T$ (an equation of state) is defined. The form of this equation of state, i.e., the definition of temperature, is a question of physical modeling which cannot be answered self-consistently in the context of a moment expansion. We can however use the same Chapman-Enskog expansion used in the derivation of Eqs. (126) - (128) to define temperature in terms of the "quantum Maxwellian" $\Omega$, by relating $w$ to the quantity $\beta$ in the Bloch equation and setting $\beta=1 / T$. This is somewhat questionable, since inherently in the definition of $e^{-\beta H}, \beta$ has to be a constant. One has to argue that the constant $\beta$ is replaced by a slowly varying function, which allows for the definition of a local Maxwellian without which the definition of a temperature in the sense of fluid dynamics would not be possible. In scaled variables the equation of state is then given by the following

Proposition 3 In the regime considered in this section the secondmoment $\omega_{2}=\operatorname{Tr} M^{2} \Omega$ is given asymptotically in terms of the zero order moment $\omega_{1}=M^{0} \Omega$ and the inverse temperature $\beta$ by the equation of state

$$
\begin{aligned}
\omega_{2}= & \frac{3 \omega_{1}}{\beta}-\frac{\lambda^{2} h^{2}}{12} \omega_{1} \beta \Delta_{x}\left\{\frac{1}{\beta} \ln \left[\omega_{1}\left(\frac{\beta}{2 \pi}\right)^{3 / 2}\right]\right\} \\
& +\left.\varepsilon A \omega_{1} \frac{h^{2} \beta}{4} \Delta_{y} \Gamma_{2}\left(\frac{h \sqrt{\beta}}{2 i} \nabla_{y}\right) V^{B}(y)\right|_{y=x / \lambda} \\
& +o\left(\lambda^{2}\right)+o(\varepsilon) .
\end{aligned}
$$

The proof of Proposition 3 is also deferred to the end of this section. If we revert to unscaled variables in Eq. (129), set $\omega_{1}=n$ and $\omega_{2}=m w$, and set the unscaled variable $\beta$ to $\beta=1 / T$, we obtain

$$
\begin{aligned}
w= & 3 n T-\frac{\hbar^{2} n}{12 m T} \Delta_{x}\left\{T \ln \left[n\left(\frac{\hbar^{2}}{2 \pi m T}\right)^{3 / 2}\right]\right\} \\
& +\frac{e \hbar^{2} n}{4 m T} \Delta_{x} \Gamma_{2}\left(\frac{\hbar}{2 i \sqrt{m T}} \nabla_{x}\right) V^{B}(x)
\end{aligned}
$$

which can be used as an equation of state expressing the internal energy density $w$ in terms of the temperature $T$. If we use Eq. (130) to express the stress tensors $P^{C}, P^{Q}$ in terms of temperature we obtain

$$
\begin{aligned}
P_{j k}^{C}= & \delta_{j k}\left\{n T+\frac{\hbar^{2} n}{36 m T} \Delta_{x}\left\{T \ln \left[\frac{1}{n}\left(\frac{2 \pi m T}{\hbar^{2}}\right)^{3 / 2}\right]\right\}\right. \\
& \left.-\frac{e \hbar^{2} n}{12 m T} \Delta_{x} \Gamma_{2}\left(\frac{\hbar}{2 i \sqrt{m T}} \nabla_{x}\right) V^{B}(x)\right\}, \quad(131)
\end{aligned}
$$




$$
\begin{aligned}
& P_{j k}^{Q 1}= \frac{n}{12 m T}\left(\partial_{x_{j k}} H-\frac{1}{3} \delta_{j k} \Delta_{x} H\right), \\
& H=T \ln \left[\frac{1}{n}\left(\frac{2 \pi m T}{\hbar^{2}}\right)^{3 / 2}\right], \\
& P_{j k}^{Q 2}=\frac{e n}{12 m T}\left\{3 \partial_{x_{j k}}-\delta_{j k} \Delta_{x}\right\} \\
& \Gamma_{2}\left(\frac{\hbar}{2 i \sqrt{m T}} \nabla_{x}\right) V^{B}(x),
\end{aligned}
$$

where we have neglected $O\left(\hbar^{4}\right)$ terms and simply replaced $w$ by $3 n T$ in Eqs. (132) and (133). Rearranging slightly and moving the $O\left(\hbar^{2}\right)$ terms from $P^{C}$ to $P^{Q}$ gives

$$
\begin{gathered}
P=n T \delta_{j k}+\hbar^{2} P^{Q 1^{\prime}}+\hbar^{2} P^{Q 2^{\prime}}, \\
P_{j k}^{Q 1^{\prime}}=\frac{n}{12 m T} \partial_{x_{j k}} H, \quad H=T \ln \left[\frac{1}{n}\left(\frac{2 \pi m T}{\hbar^{2}}\right)^{3 / 2}\right],
\end{gathered}
$$

$$
P_{j k}^{Q 2^{\prime}}=\frac{e n}{4 m T} \partial_{x_{j k}} \Gamma_{2}\left(\frac{\hbar}{2 i \sqrt{m T}} \nabla_{x}\right) V^{B}(x) .
$$

We close the paper by proving Propositions 2 and 3 via the Chapman-Enskog expansion of the equilibrium distribution function.

Proof of Proposition 2 Setting $\varepsilon=0$ in Eq. (102) gives for the zero order term

$$
\begin{gathered}
\partial_{\beta} \Omega_{0}(x, p, \beta)=\frac{\lambda^{2} h^{2}}{8} \Delta_{x} \Omega_{0}-\frac{1}{2}|p|^{2} \Omega_{0}-\Theta[U] \Omega_{0}, \\
\Omega_{0}(x, p, \beta=0)=1, \\
\Theta[U]=\frac{A}{2}\left[U\left(x+\frac{h \lambda}{2 i} \nabla_{p}\right)+U\left(x-\frac{h \lambda}{2 i} \nabla_{p}\right)\right] .
\end{gathered}
$$

Since the potential $U$ is assumed to be sufficiently smooth the operator $\Theta$ can be approximated by Taylor expansion in $\lambda$ giving

$$
\Theta[U]=A U(x)-\frac{\lambda^{2} h^{2} A}{8} \sum_{\mu \nu} \partial_{x_{\mu \nu}}^{2} U \partial_{p_{\mu \nu}}^{2}+O\left(\lambda^{4}\right) .
$$

The solution of Eq. (137) can be computed asymptotically by setting $\lambda=0$. For the zero order term we obtain

$$
\begin{gathered}
\partial_{\beta} \Omega_{00}(x, p, \beta)=-\frac{1}{2}|p|^{2} \Omega_{00}-A U \Omega_{00} \\
\Omega_{00}(x, p, \beta=0)=1,
\end{gathered}
$$

giving in zero order the classical Maxwellian

$$
\begin{aligned}
& \Omega_{00}(x, p, \beta)=\exp \left[-\frac{\beta}{2}|p|^{2}-A \beta U\right], \\
& \omega_{1}^{00}=M^{0} \Omega_{00}=\left(\frac{2 \pi}{\beta}\right)^{3 / 2} \exp [-A \beta U], \\
& \omega_{2}^{00}=\operatorname{Tr} M^{2} \Omega_{00}=\frac{3}{\beta}\left(\frac{2 \pi}{\beta}\right)^{3 / 2} \exp [-A \beta U] .
\end{aligned}
$$

This lets us express $U$ and $\beta$ in terms of the moment vector $\omega$ as

$$
\beta=\frac{3 \omega_{1}^{00}}{\omega_{2}^{00}}, A U=\frac{\omega_{2}^{00}}{3 \omega_{1}^{00}}\left[-\ln \omega_{1}^{00}-\frac{3}{2} \ln \left(\frac{3 \omega_{1}^{00}}{2 \pi \omega_{2}^{00}}\right)\right],
$$

and from now on the potential $U$ and the inverse temperature $\beta$ are given in terms of the parameter $\omega^{00}$. Thus we obtain the zero order shape function $\phi_{00}$ as

$$
\phi_{00}(\omega, p)=\omega_{1}\left(\frac{3 \omega_{1}}{2 \pi \omega_{2}}\right)^{3 / 2} \exp \left(-\frac{3 \omega_{1}}{2 \omega_{2}}|p|^{2}\right)
$$

For the next term in the expansion of $\Omega_{0}$ we obtain the initial value problem

$$
\begin{aligned}
& \partial_{\beta} \Omega_{01}(x, p, \beta) \\
& =\frac{h^{2}}{8} \Delta_{x} \Omega_{00}-\frac{1}{2}|p|^{2} \Omega_{01}-A U(x) \Omega_{01} \\
& +\frac{h^{2} A}{8} \sum_{\mu \nu}\left(\partial_{x_{\mu \nu}}^{2} U\right) \partial_{p_{\mu \nu}}^{2} \Omega_{00} \\
& \Omega_{01}(x, p, \beta=0)=0 .
\end{aligned}
$$


Using $\Omega_{00}$ as an integrating factor in Eq. (145) we obtain

$$
\begin{gathered}
\Omega_{01}(x, p, \beta)=\Omega_{00}(x, p, \beta) \int_{0}^{\beta} \frac{1}{\Omega_{00}(x, p, \gamma)} \\
\quad \frac{h^{2}}{8} \Delta_{x} \Omega_{00}(x, p, \gamma) \\
\left.+\frac{h^{2} A}{8} \sum_{\mu \nu}\left(\partial_{x_{\mu \nu}}^{2} U\right) \partial_{p_{\mu \nu}}^{2} \Omega_{00}(x, p, \gamma)\right] d \gamma \\
=\frac{h^{2} A}{8} \Omega_{00}(x, p, \beta) \int_{0}^{\beta}\left[-2 \gamma \Delta_{x} U+A \gamma^{2}\left|\nabla_{x} U\right|^{2}\right. \\
\left.+\sum_{\mu \nu}\left(\partial_{x_{\mu \nu}}^{2} U\right) \gamma^{2} p_{\mu} p_{\nu}\right] d \gamma \\
=\frac{h^{2} A}{8} \Omega_{00}(x, p, \beta)\left[-\beta^{2} \Delta_{x} U+\frac{A \beta^{3}}{3}\left|\nabla_{x} U\right|^{2}\right. \\
\left.+\sum_{\mu \nu}\left(\partial_{x_{\mu \nu}}^{2} U\right) \frac{\beta^{3}}{3} p_{\mu} p_{\nu}\right] .
\end{gathered}
$$

In order to obtain the first order term $\phi_{01}$ in the Chapman-Enskog expansion of $\Omega$ we have to write

$$
\begin{aligned}
\left(\Omega_{00}\right. & \left.+\lambda^{2} \Omega_{01}\right)(x, p, \beta) \\
& =\left(\phi_{00}+\lambda^{2} \phi_{01}\right)\left(\omega^{00}+\lambda^{2} \omega^{01}, p\right),
\end{aligned}
$$

where $\omega^{01}$ is the moment vector of $\Omega_{01}$. Expanding Eq. (148) up to order $O\left(\lambda^{2}\right)$ gives

$$
\phi_{01}\left(\omega^{00}, p\right)=\Omega_{01}(x, p, \beta)-\sum_{j} \frac{\partial \phi^{00}}{\partial \omega_{j}}\left(\omega^{00}, p\right) \omega_{j}^{01} .
$$

If we express $\Omega_{01}$ and its moments $\omega^{01}$ in terms of $\omega^{00}$ we obtain the right-hand side of Eq. (149). In order to simplify the calculation we first rewrite $\Omega_{01}$ separating the terms depending explicitly on $p$ from the others. We write $\Omega_{01}$ as

$$
\begin{aligned}
& \Omega_{01}(x, p, \beta)=K^{0} G^{0}+\sum_{\mu \nu} K_{\mu, \nu}^{1} G_{\mu, \nu}^{1}, \\
& K^{0}=\frac{h^{2}}{24} \omega_{1}^{00}\left(-3 \beta^{2} \Delta_{x} H+\beta^{3}\left|\nabla_{x} H\right|^{2}\right), \\
& G^{0}=\left(\frac{\beta}{2 \pi}\right)^{3 / 2} \exp \left(-\frac{\beta}{2}|p|^{2}\right),
\end{aligned}
$$

$$
\begin{aligned}
K_{\mu, \nu}^{1} & =\frac{h^{2}}{24} \omega_{1}^{00} \beta^{3}\left(\partial_{x_{\mu \nu}}^{2} H\right), \\
G_{\mu \nu}^{1} & =\left(\frac{\beta}{2 \pi}\right)^{3 / 2} \exp \left(-\frac{\beta}{2}|p|^{2}\right) p_{\mu} p_{\nu}, \\
H=A U & =\frac{1}{\beta}\left[-\ln \omega_{1}^{00}-\frac{3}{2} \ln \left(\frac{\beta}{2 \pi}\right)\right], \beta=\frac{3 \omega_{1}^{00}}{\omega_{2}^{00}} .
\end{aligned}
$$

Introducing the vector $\kappa=\left(1,|p|^{2}\right)$, the moments $\omega^{01}$ are now given by

$$
\begin{gathered}
\omega_{j}^{01}=\int \kappa_{j} \Omega^{01} d p=K^{0} q_{j}+\sum_{\mu \nu} K_{\mu \nu}^{1} R_{j \mu \nu}, \\
q_{j}=\int \kappa_{j} G^{0} d p, \quad R_{j \mu \nu}=\int \kappa_{j} G_{\mu \nu}^{1} d p
\end{gathered}
$$

and from Eq. (149) the first order shape function $\phi_{01}$ is given by

$$
\begin{aligned}
\phi_{01}\left(\omega^{00}, p\right)= & K_{0}\left[G^{0}-\sum_{j} \frac{\partial \phi^{00}}{\partial \omega_{j}}\left(\omega^{00}\right) q_{j}\right] \\
& +\sum_{\mu \nu} K_{\mu \nu}^{1}\left[G_{\mu \nu}^{1}-\sum_{j} \frac{\partial \phi^{00}}{\partial \omega_{j}}\left(\omega^{00}\right) R_{j \mu \nu}\right]
\end{aligned}
$$

Computing the moments $q_{j}$ and $R_{j \mu \nu}$ gives

$q_{1}=1, q_{2}=\frac{3}{\beta}, R_{1 \mu \nu}=\frac{\delta_{\mu \nu}}{\beta}, R_{2 \mu \nu}=\frac{5 \delta_{\mu \nu}}{\beta^{2}}$,

and for the derivatives of $\phi_{00}$ with respect to $\omega$ we obtain

$$
\begin{aligned}
\frac{\partial \phi_{00}}{\partial \omega_{1}}\left(\omega^{00}\right)= & \left(\frac{\beta}{2 \pi}\right)^{3 / 2} \exp \left(-\frac{\beta}{2}|p|^{2}\right) \\
& {\left[\frac{5}{2}-\frac{\beta}{2}|p|^{2}\right], } \\
\frac{\partial \phi_{00}}{\partial \omega_{2}}\left(\omega^{00}\right)= & \frac{1}{3}\left(\frac{\beta}{2 \pi}\right)^{3 / 2} \exp \left(-\frac{\beta}{2}|p|^{2}\right) \\
& {\left[-\frac{3 \beta}{2}+\frac{\beta^{2}}{2}|p|^{2}\right] . }
\end{aligned}
$$


Inserting Eqs. (156) -(158) into Eq. (155) gives

$$
\begin{gathered}
\phi_{01}(\omega, p)=\frac{h^{2}}{24} \omega_{1}\left(\frac{\theta}{2 \pi}\right)^{3 / 2} \exp \left(-\frac{\theta}{2}|p|^{2}\right) \theta^{3} \\
\sum_{\mu \nu}\left(\partial_{x_{\mu \nu}}^{2} H\right)\left[p_{\mu} p_{\nu}-\frac{\delta_{\mu \nu}}{3}|p|^{2}\right], \\
H=\frac{1}{\theta}\left[-\ln \omega_{1}-\frac{3}{2} \ln \left(\frac{\theta}{2 \pi}\right)\right], \theta=\frac{3 \omega_{1}}{\omega_{2}} .
\end{gathered}
$$

We now turn our attention to the first order term $\Omega_{1}$ in $\varepsilon . \Omega_{1}$ satisfies the initial value problem

$$
\begin{array}{r}
\partial_{\beta} \Omega_{1}(x, p, \beta)=\frac{\lambda^{2} h^{2}}{8} \Delta_{x} \Omega_{1}-\frac{1}{2}|p|^{2} \Omega_{1}-\Theta[U] \Omega_{1} \\
-\Theta\left[V^{B}\right] \Omega_{0}, \quad \Omega_{1}(x, p, \beta=0)=0, \\
\Theta[U]=\frac{A}{2}\left[U\left(x+\frac{h \lambda}{2 i} \nabla_{p}\right)+U\left(x-\frac{h \lambda}{2 i} \nabla_{p}\right)\right], \\
\Theta\left[V^{B}\right]=\frac{A}{2}\left[V^{B}\left(\frac{x}{\lambda}+\frac{h}{2 i} \nabla_{p}\right)+V^{B}\left(\frac{x}{\lambda}-\frac{h}{2 i} \nabla_{p}\right)\right] .
\end{array}
$$

On this spatial scale $\Omega_{1}$ will exhibit rapid variation near $x=0$ because of the dependence of $V^{B}$ on $x / \lambda$ in Eq. (163). It is therefore necessary to rescale $x$ and write the first order term as $\Omega_{1}=\Omega_{1}(x / \lambda, p, \beta)$. Written in the rescaled spatial variable $y=x / \lambda$, Eqs. (161)-(163) read

$$
\begin{aligned}
\partial_{\beta} \Omega_{1}(y, p, \beta)= & \frac{h^{2}}{8} \Delta_{y} \Omega_{1}-\frac{1}{2}|p|^{2} \Omega_{1}-\Theta[U] \Omega_{1} \\
& -\Theta\left[V^{B}\right] \Omega_{0}, \Omega_{1}(y, p, \beta=0)=0 \\
\Theta[U] \Omega_{1}=\frac{A}{2}[ & U\left(\lambda y+\frac{h \lambda}{2 i} \nabla_{p}\right) \\
& \left.+U\left(\lambda y-\frac{h \lambda}{2 i} \nabla_{p}\right)\right] \Omega_{1}(y, p, \beta),(165) \\
\Theta\left[V^{B}\right] \Omega_{0}=\frac{A}{2}[ & V^{B}\left(y+\frac{h}{2 i} \nabla_{p}\right) \\
& \left.+V^{B}\left(y-\frac{h}{2 i} \nabla_{p}\right)\right] \Omega_{0}(\lambda y, p, \beta) .
\end{aligned}
$$

To compute the zero order term in $\lambda$ of Eq. (164), i.e., to compute $\Omega_{10}$, we set $\lambda=0$ and obtain

$$
\begin{aligned}
\partial_{\beta} \Omega_{10}(y, p, \beta)= & \frac{h^{2}}{8} \Delta_{y} \Omega_{10}-\frac{1}{2}|p|^{2} \Omega_{10} \\
& -A U(0) \Omega_{10}-\Theta\left[V^{B}\right] \Omega_{00}, \\
\Theta\left[V^{B}\right] \Omega_{00}=\frac{A}{2}[ & V^{B}\left(y+\frac{h}{2 i} \nabla_{p}\right) \\
& \left.+V^{B}\left(y-\frac{h}{2 i} \nabla_{p}\right)\right] \Omega_{00}(0, p, \beta) .
\end{aligned}
$$

Because of the Maxwellian form of $\Omega_{00}$ the term $\Theta\left[V^{B}\right] \Omega_{00}$ can be computed exactly in terms of the spatial Fourier transform of the barrier potential $V^{B}$. Using the definition of the pseudo-differential operator $\Theta\left[V^{B}\right]$ we obtain

$$
\begin{gathered}
V^{B}\left(y+\frac{h}{2 i} \nabla_{p}\right) \Omega_{00}(0, p, \beta)= \\
(2 \pi)^{-3 / 2} \int V^{B}\left(y+\frac{h}{2} \eta\right) \hat{\Omega}_{00}(0, \eta, \beta) \\
\exp (i \eta \cdot p) d \eta,
\end{gathered}
$$

where $\hat{\Omega}_{00}(0, \eta, \beta$.) is, because of Eq. (141), given by $\hat{\Omega}_{00}(0, \eta, \beta)$

$$
\begin{aligned}
& =\left(\frac{1}{2 \pi}\right)^{3 / 2} \int \exp \left(-A \beta U(0)-\frac{\beta}{2}|p|^{2}-i \eta \cdot p\right) d p \\
& =\left(\frac{1}{2 \pi}\right)^{3 / 2} \omega_{1}^{00}(0) \exp \left(-\frac{|\eta|^{2}}{2 \beta}\right) .
\end{aligned}
$$

Inserting Eq. (170) into Eq. (169) yields

$$
\begin{aligned}
& V^{B}\left(y+\frac{h}{2 i} \nabla_{p}\right) \Omega_{00}(0, p, \beta) \\
& =\exp \left(-A \beta U(0)-\frac{\beta}{2}\left|p+\frac{h}{2 i} \nabla_{y}\right|^{2}\right) V^{B}(y), \\
& \Theta\left[V^{B}\right] \Omega_{00}(0, p, \beta) \\
& =A \exp (-A \beta U(0)) G\left(p, \frac{h}{i} \nabla_{y}\right) V^{B}(y), \\
& G(p, \xi)=\frac{1}{2}\left[\exp \left(-\frac{\beta}{2}\left|p+\frac{1}{2} \xi\right|^{2}\right)\right. \\
& \left.\quad+\exp \left(-\frac{\beta}{2}\left|p-\frac{1}{2} \xi\right|^{2}\right)\right]
\end{aligned}
$$


Equation (167) can now be solved by using the Green's function of the Laplace operator or, equivalently, by simply Fourier transforming the equation in the spatial variable $y$. Denoting the Fourier transform of $\Omega_{10}$ with $g$ and the Fourier transform of $V_{B}$ with $Z$, this gives the initial value problem

$$
\begin{gathered}
\partial_{\beta} g(\xi, p, \beta)=-\frac{h^{2}|\xi|^{2}}{8} g-\frac{1}{2}|p|^{2} g-A U(0) g \\
-A \exp (-A \beta U(0)) G(p, h \xi) Z(\xi), \\
g(\xi, p, \beta=0)=0
\end{gathered}
$$

which has as a solution

$$
\begin{aligned}
g(\xi, p, \beta)= & -\frac{2 A}{h p \cdot \xi} \sinh \left(\frac{\beta h}{2} p \cdot \xi\right) \\
& \exp \left[-\frac{\beta h^{2}|\xi|^{2}}{8}-\frac{\beta}{2}|p|^{2}-\beta A U(0)\right] Z(\xi) .
\end{aligned}
$$

After back Fourier transformation $\Omega_{10}$ can be written as a smoothing operator acting on the barrier potential $V^{B}$. Expressing the exponential of $U$ in terms of the zero order moment $\omega_{1}^{00}$, this gives

$$
\begin{gathered}
\Omega_{10}(y, p, \beta)=-A\left(\frac{\beta}{2 \pi}\right)^{3 / 2} \omega_{1}^{00}(0) \\
\beta S\left(\sqrt{\beta} p, \frac{h \sqrt{\beta}}{2 i} \nabla_{y}\right) V^{B}(y), \quad(177) \\
S(p, \xi)=\frac{1}{p \cdot \xi} \sinh (p \cdot \xi) \exp \left[-\frac{1}{2}|\xi|^{2}-\frac{1}{2}|p|^{2}\right] .
\end{gathered}
$$

$S$ is a smoothing operator because of the decay of the symbol in the frequency variable $\xi$. Proceeding in the same way as before, we obtain the expression for the shape function $\phi_{01}$ from expanding the equation

$$
\begin{aligned}
\left(\Omega_{00}+\right. & \left.\lambda^{2} \Omega_{01}+\varepsilon \Omega_{10}\right)(x, p, \beta)+\cdots \\
= & \left(\phi_{00}+\lambda^{2} \phi_{01}+\varepsilon \phi_{10}\right) \\
& \left(\omega^{00}+\lambda^{2} \omega^{01}+\varepsilon \omega^{10}+\cdots, p, V^{B}\right)+\cdots
\end{aligned}
$$

in powers of $\lambda$ and $\varepsilon$. As before, this gives

$$
\begin{aligned}
\phi_{10}\left(\omega^{00}, p, V^{B}\right)= & \Omega_{10}(x, p, \beta) \\
& -\sum_{j} \frac{\partial \phi_{00}}{\partial \omega_{j}}\left(\omega^{00}, p\right) \omega_{j}^{10} .
\end{aligned}
$$

Again we separate $\Omega_{10}$ into terms depending on $p$ and the rest, writing $\Omega_{10}$ as

$$
\begin{aligned}
\Omega_{10} & =-K S\left(\sqrt{\beta} p, \frac{h \sqrt{\beta}}{2 i} \nabla_{y}\right) V^{B}(y), \\
K & =A \beta\left(\frac{\beta}{2 \pi}\right)^{3 / 2} \omega_{1}^{00}(0),
\end{aligned}
$$

yielding for the moments:

$$
\begin{gathered}
\omega_{1}^{10}=-K\left(\frac{2 \pi}{\beta}\right)^{3 / 2} \Gamma_{0}\left(\frac{h \sqrt{\beta}}{2 i} \nabla_{y}\right) V^{B}(y), \\
\omega_{2}^{10}=-K \beta^{-5 / 2}(2 \pi)^{3 / 2} \Gamma_{1}\left(\frac{h \sqrt{\beta}}{2 i} \nabla_{y}\right) V^{B}(y), \\
\Gamma_{0}(\xi)=\left(\frac{1}{2 \pi}\right)^{3 / 2} \int S(p, \xi) d p \\
\Gamma_{1}(\xi)=\left(\frac{1}{2 \pi}\right)^{3 / 2} \int|p|^{2} S(p, \xi) d p
\end{gathered}
$$

Inserting this into Eq. (180) gives

$$
\begin{aligned}
& \phi_{10}\left(\omega^{00}, p, V^{B}\right) \\
& =\frac{1}{2} A \beta\left(\frac{\beta}{2 \pi}\right)^{3 / 2} \\
& \omega_{1}^{00}(0)\left\{-2 S\left(\sqrt{\beta} p, \frac{h \sqrt{\beta}}{2 i} \nabla_{y}\right)+\exp \left(-\frac{\beta}{2}|p|^{2}\right),\right. \\
& \quad\left[\left(5 \Gamma_{0}-\Gamma_{1}\right)\left(\frac{h \sqrt{\beta}}{2 i} \nabla_{y}\right)\right. \\
& \left.\left.+\beta|p|^{2}\left(\frac{1}{3} \Gamma_{1}-\Gamma_{0}\right)\left(\frac{h \sqrt{\beta}}{2 i} \nabla_{y}\right)\right]\right\} V^{B}(y), \\
& \beta:=\frac{3 \omega_{1}^{00}}{\omega_{2}^{00} .} \quad(184),(185)
\end{aligned}
$$


Replacing $y$ by $x / \lambda$, we obtain for $\phi_{01}$ :

$$
\begin{aligned}
& \phi_{10}\left(\omega, p, V^{B}\right)= \frac{1}{2} A \theta\left(\frac{\theta}{2 \pi}\right)^{3 / 2} \\
& \omega_{1}\{-2 S\left(\sqrt{\theta} p, \frac{h \sqrt{\theta}}{2 i} \nabla_{y}\right) \\
&+ \exp \left(-\frac{\theta}{2}|p|^{2}\right), \\
& {\left[\left(5 \Gamma_{0}-\Gamma_{1}\right)\left(\frac{h \sqrt{\theta}}{2 i} \nabla_{y}\right)\right.} \\
&+ \theta|p|^{2}\left(\frac{1}{3} \Gamma_{1}-\Gamma_{0}\right) \\
&\left.\left.\left(\frac{h \sqrt{\theta}}{2 i} \nabla_{y}\right)\right]\right\}\left.V^{B}(y)\right|_{y=\frac{x}{\lambda}} \\
& \theta:=\frac{3 \omega_{1}}{\omega_{2}}, \quad(186)
\end{aligned}
$$

where we committed an $O(\lambda)$ error in replacing $\omega_{1}^{00}(0)$ by $\omega_{1}^{00}(\lambda y)$ in Eq. (181).

It remains to compute the moments of the smoothing operator $S$, i.e., the operators $\Gamma_{0}$ and $\Gamma_{1}$. Calculating moments of the symbol $S$ is actually easier if it is written in a slightly different form. Using the fact that $(\partial / \partial \gamma)[(1 / p \cdot \xi) \sinh$ $(\gamma p \cdot \xi)]=\cosh (\gamma p \cdot \xi)$, we obtain

$$
\begin{aligned}
& S(p, \xi)= \int_{0}^{1} \cosh (\gamma p \cdot \xi) \\
& \exp \left[-\frac{1}{2}|\xi|^{2}-\frac{1}{2}|p|^{2}\right] d \gamma \\
&=\frac{1}{2} \int_{0}^{1} \exp \left[\frac{|\xi|^{2}}{2}\left(\gamma^{2}-1\right)\right] \\
& {\left[\exp \left(-\frac{1}{2}|p+\gamma \xi|^{2}\right)\right.} \\
&\left.+\exp \left(-\frac{1}{2}|p-\gamma \xi|^{2}\right)\right] d \gamma
\end{aligned}
$$

giving for the moments of $S$ :

$$
\begin{aligned}
\Gamma_{0}(\xi) & =(2 \pi)^{-3 / 2} \int S(p, \xi) d p \\
& =\int_{0}^{1} \exp \left[\frac{|\xi|^{2}}{2}\left(\gamma^{2}-1\right)\right] d \gamma
\end{aligned}
$$

$$
\begin{aligned}
& \int p_{\mu} p_{\nu} S(p, \xi) d p=(2 \pi)^{3 / 2} \int_{0}^{1}\left[\delta_{\mu \nu}+\gamma^{2} \xi_{\mu} \xi_{\nu}\right] \\
& \exp \left[\frac{|\xi|^{2}}{2}\left(\gamma^{2}-1\right)\right] d \gamma \\
&=(2 \pi)^{3 / 2}\left[\delta_{\mu \nu} \Gamma_{0}(\xi)+\frac{1}{3} \xi_{\mu} \xi_{\nu} \Gamma_{2}(\xi)\right] \\
& \Gamma_{2}(\xi)=\int_{0}^{1} \gamma^{2} \exp \left[\frac{|\xi|^{2}}{2}\left(\gamma^{2}-1\right)\right] d \gamma
\end{aligned}
$$

and therefore $\Gamma_{1}(\xi)=3 \Gamma_{0}(\xi)+|\xi|^{2} \Gamma_{2}(\xi)$.

In summary, the approximate solution of the Bloch equation (102) can be written as a function of its moments and the barrier potential as

$$
\begin{aligned}
\Omega(x, p, \beta)= & \phi_{00}(\omega, p)+\lambda^{2} \phi_{01}(\omega, p) \\
& +\varepsilon \phi_{10}\left(\omega, p, V^{B}\right)+o\left(\lambda^{2}\right)+o(\varepsilon),
\end{aligned}
$$

$$
\begin{gathered}
\omega=\int\left(1,|p|^{2}\right) \Omega(x, p, \beta) d p=\left(M^{0}, t r M^{2}\right) \Omega, \\
\phi_{00}(\omega, p)=\omega_{1}\left(\frac{\theta}{2 \pi}\right)^{3 / 2} \exp \left(-\frac{\theta}{2}|p|^{2}\right), \\
\phi_{01}(\omega, p)=\frac{h^{2}}{24} \omega_{1}\left(\frac{\theta}{2 \pi}\right)^{3 / 2} \exp \left(-\frac{\theta}{2}|p|^{2}\right) \theta^{3} \\
\sum_{\mu \nu}\left(\partial_{x_{\mu \nu}} H\right)\left[p_{\mu} p_{\nu}-\frac{\delta_{\mu \nu}}{3}|p|^{2}\right], \\
\begin{aligned}
\phi_{10}\left(\omega, p, V^{B}\right)=\frac{1}{2} A \theta\left(\frac{\theta}{2 \pi}\right)^{3 / 2} \omega_{1} \\
\left\{-2 S\left(\sqrt{\theta} p, \frac{h \sqrt{\theta}}{2 i} \nabla_{y}\right)\right. \\
+\exp \left(-\frac{\theta}{2}|p|^{2}\right) \\
{\left[\left(2 \Gamma_{0}+\frac{\theta h^{2}}{4} \Delta_{y} \Gamma_{2}\right)\right.} \\
\left(\frac{h \sqrt{\theta}}{2 i} \nabla_{y}\right)-\frac{h^{2} \theta^{2}}{12}|p|^{2} \Delta_{y} \Gamma_{2} \\
\left.\left.\left(\frac{h \sqrt{\theta}}{2 i} \nabla_{y}\right)\right]\right\}\left.V^{B}(y)\right|_{y=x / \lambda}
\end{aligned}
\end{gathered}
$$




$$
H=\frac{1}{\theta}\left[-\ln \omega_{1}-\frac{3}{2} \ln \left(\frac{\theta}{2 \pi}\right)\right], \quad \theta=\frac{3 \omega_{1}}{\omega_{2}},
$$

where the dependence of $\Omega$ on the barrier potential $V^{B}$ is of a functional form involving the pseudodifferential operators $S, \Gamma_{0}$, and $\Gamma_{1}$ defined in Eqs. (178) and (183).

Proof of Proposition 3 From the Chapman-Enskog expansion in the preceding proof the parameter $\omega_{2}=\operatorname{Tr} M^{2} \Omega=\omega_{2}^{00}+\lambda^{2} \omega_{2}^{01}+\varepsilon \omega_{2}^{10}$ can be expressed asymptotically in terms of $\beta$ and the zero order moment $\omega_{1}=\operatorname{Tr} M^{0} \Omega=\omega_{1}^{00}+\lambda^{2} \omega_{1}^{01}+$ $\varepsilon \omega_{1}^{10}$ as

$$
\begin{aligned}
\omega_{2}= & g_{00}\left(\omega_{1}, \beta\right)+\lambda^{2} g_{01}\left(\omega_{1}, \beta\right) \\
& +\varepsilon g_{10}\left(\omega_{1}, \beta, V^{B}\right)+o\left(\lambda^{2}\right)+o(\varepsilon) .
\end{aligned}
$$

Expanding the left- and right-hand sides gives for the functions $g_{00}, g_{01}$, and $g_{10}$

$$
\begin{aligned}
& \omega_{2}^{00}= g_{00}\left(\omega_{1}^{00}, \beta\right) \\
& \omega_{2}^{01}= \partial_{\omega_{1}} g_{00}\left(\omega_{1}^{00}, \beta\right) \omega_{1}^{01} \\
&+g_{01}\left(\omega_{1}^{00}, \beta\right) \\
& \omega_{2}^{10}=\partial_{\omega_{1}} g_{00}\left(\omega_{1}^{00}, \beta\right) \omega_{1}^{10}+g_{10}\left(\omega_{1}^{00}, \beta\right) .
\end{aligned}
$$

All the moments $\omega$ in Eqs. (199), (200) have already been computed. Thus we essentially have only to read the result of the previous expansion backwards. From the proof of Proposition 2 we have

$$
\begin{gathered}
\omega_{1}^{00}=\left(\frac{2 \pi}{\beta}\right)^{3 / 2} \exp [-A \beta U], \quad \omega_{2}^{00}=\frac{3 \omega_{1}^{00}}{\beta} \\
\omega_{1}^{01}=\frac{h^{2} A}{8} \omega_{1}^{00}\left[-\frac{2 \beta^{2}}{3} \Delta_{x} U+\frac{A \beta^{3}}{3}\left|\nabla_{x} U\right|^{2}\right] \\
\omega_{2}^{01}=\frac{h^{2} A}{8} \omega_{1}^{00}\left[-\frac{4 \beta}{3} \Delta_{x} U+A \beta^{2}\left|\nabla_{x} U\right|^{2}\right], \\
\omega_{1}^{10}=-\left.A \beta \omega_{1}^{00} \Gamma_{0}\left(\frac{h \sqrt{\beta}}{2 i} \nabla_{y}\right) V^{B}(y)\right|_{y=x / \lambda},
\end{gathered}
$$

$$
\begin{gathered}
\omega_{2}^{10}=-A \omega_{1}^{00}\left[3 \Gamma_{0}-\frac{h^{2} \beta}{4} \Delta_{y} \Gamma_{2}\right] \\
\left.\left(\frac{h \sqrt{\beta}}{2 i} \nabla_{y}\right) V^{B}(y)\right|_{y=x / \lambda} .
\end{gathered}
$$

Inserting Eqs. (201)-(205) into Eqs. (199), (200) gives

$$
\begin{gathered}
g_{00}\left(\omega_{1}, \beta\right)=\frac{3 \omega_{1}}{\beta} \\
g_{01}\left(\omega_{1}, \beta\right)=-\frac{h^{2}}{12} \omega_{1} \beta \Delta_{x}\left\{\frac{1}{\beta} \ln \left[\omega_{1}\left(\frac{\beta}{2 \pi}\right)^{3 / 2}\right]\right\} \\
g_{10}\left(\omega_{1}, \beta, V^{B}\right)=A \omega_{1} \frac{h^{2} \beta}{4} \Delta_{y} \Gamma_{2} \\
\left.\left(\frac{h \sqrt{\beta}}{2 i} \nabla_{y}\right) V^{B}(y)\right|_{y=x / \lambda}
\end{gathered}
$$

which proves Proposition 3.

\section{References}

[1] Baccarani, G. and Wordeman, M. R. (1985). "An investigation of steady-state velocity overshoot effects in $\mathrm{Si}$ and GaAs devices", Solid State Electronics, 28, 407-416.

[2] Gardner, C. L. (1994). "The quantum hydrodynamic model for semiconductor devices", SIAM Journal on Applied Mathematics, 54, 409-427.

[3] Ringhofer, C. (1997). "Computational methods for semiclassical and quantum transport in semiconductor devices", Acta Numerica, 3, 485-521.

[4] Gardner, C. L. (1995). "Resonant tunneling in the quantum hydrodynamic model", VLSI Design, 3, 201-210.

[5] Gardner, C. L. and Ringhofer, C. (1996). "Smooth quantum potential for the hydrodynamic model", Physical Review, E53, 157-167.

[6] Gardner, C. L. and Ringhofer, C. (1998). "Approximation of thermal equilibrium for quantum gases with discontinuous potentials and application to semiconductor devices", SIAM Journal on Applied Mathematics, 58, 780-805.

[7] Barker, J. and Ferry, D. K. (1979). "Self-scattering pathvariable formulation of high-field, time-dependent, quantum kinetic equations for semiconductor transport in the finite collision-duration regime", Physical Review Letters, 42, 1779-1781.

[8] Argyres, P. (1992). "Quantum kinetic equations for electrons in high electric and phonon field", Physics Letters, A171.

[9] Fromlet, F., Markowich, P. and Ringhofer, C. (1999). “A Wignerfunction approach to phonon scattering", VLSI Design, 9, 339-350.

[10] Wigner, E. (1932). "On the quantum correction for thermodynamic equilibrium”, Physical Review, 40, 749-759. 


\section{Authors' Biographies}

Carl L. Gardner is Professor of Mathematics at Arizona State University. His current research interests lie in classical and quantum semiconductor device simulation, computational fluid dynamics, astrophysical flows, and the modeling and simulation of ion transport in the channels of cellular membranes.

Christian Ringhofer is Professor of Mathematics at Arizona State University. His current research interests include classical and quantum transport equations and moment models for semiconductor device modeling. 

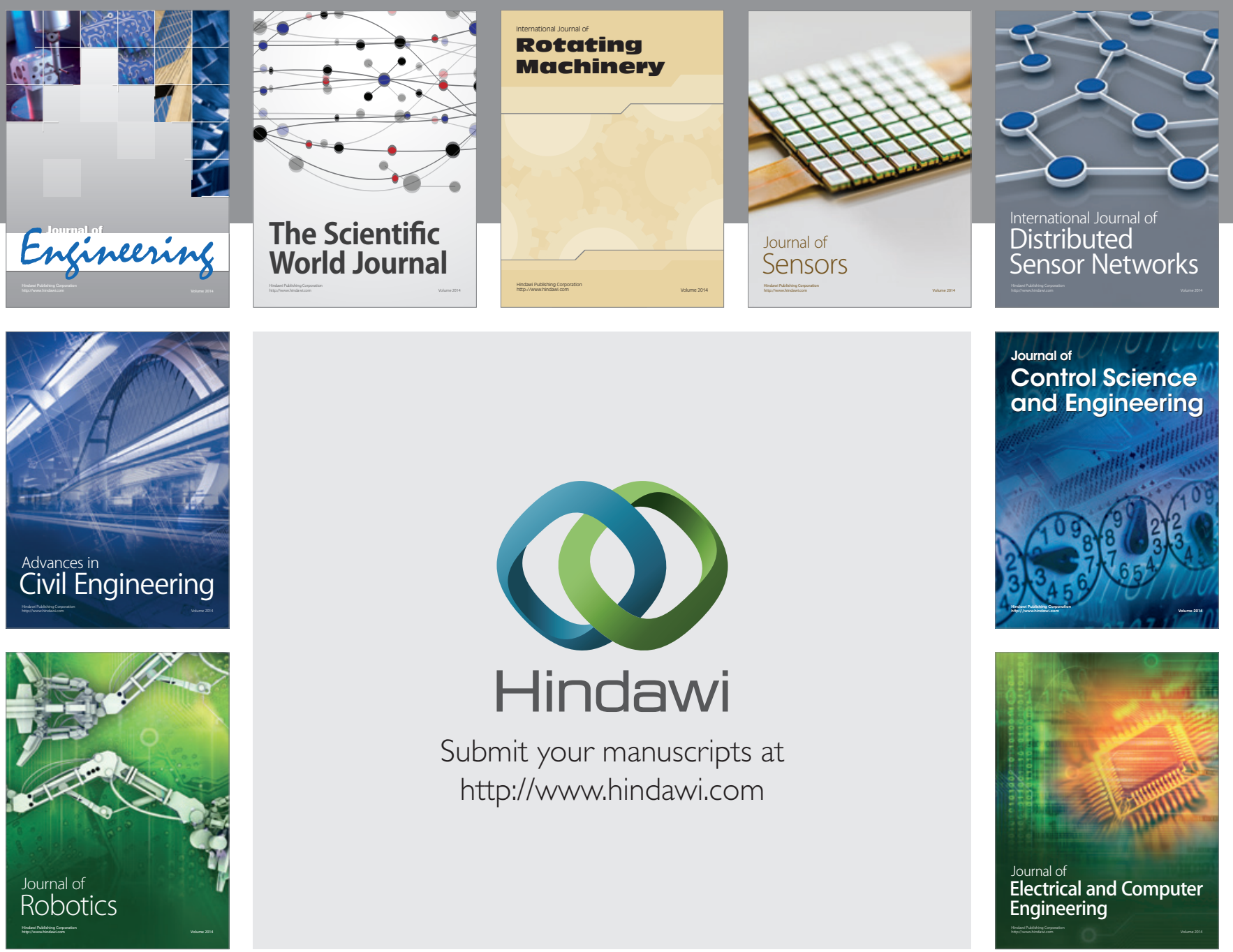

Submit your manuscripts at

http://www.hindawi.com
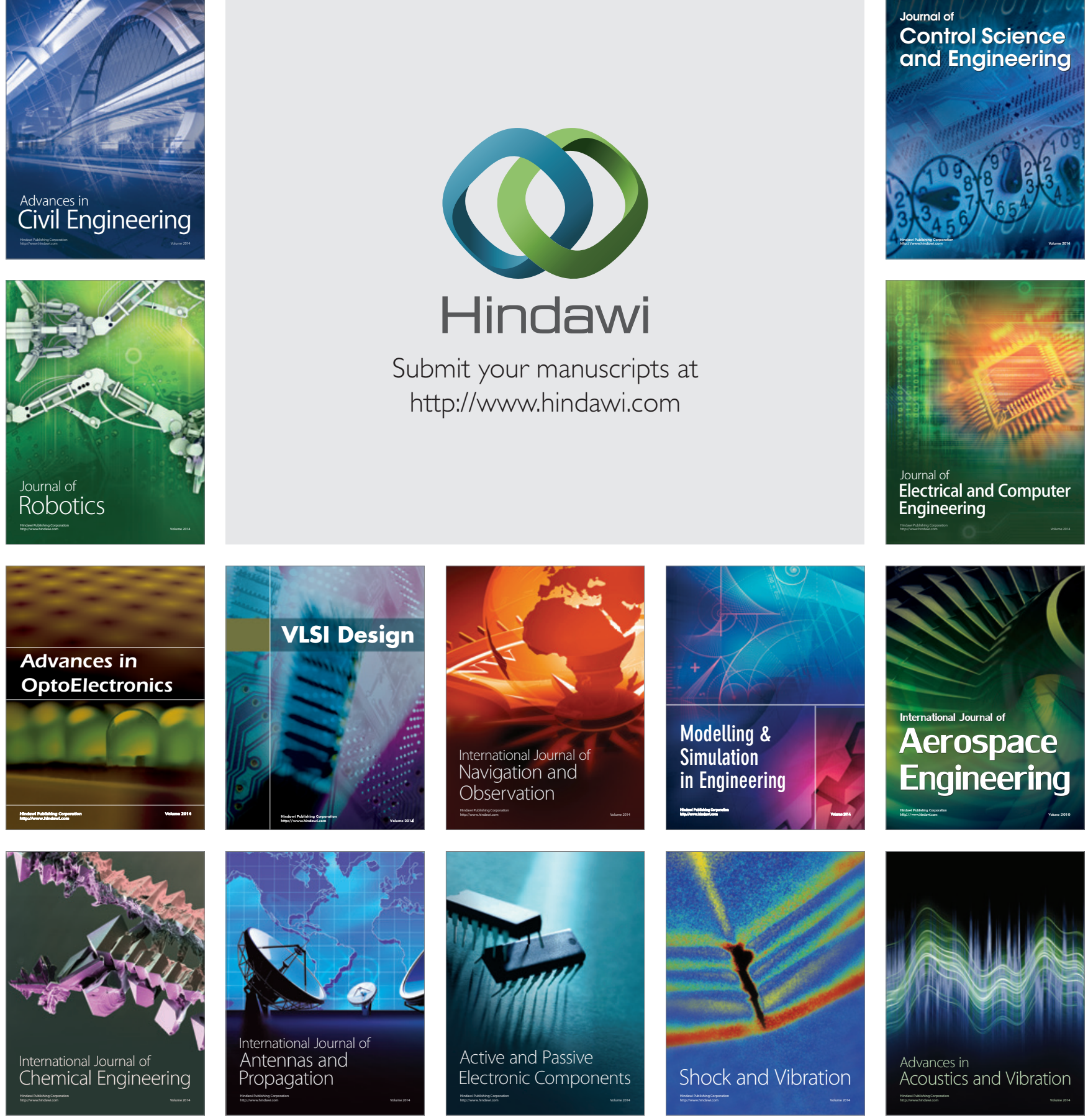